TITLE:

\title{
Invariant Manifolds Around Soliton Manifolds for the Nonlinear Klein-Gordon Equation
}

$\operatorname{AUTHOR}(\mathrm{S}):$

Nakanishi, K.; Schlag, W.

\section{CITATION:}

Nakanishi, K.... [et al]. Invariant Manifolds Around Soliton Manifolds for the Nonlinear Klein-Gordon Equation. SIAM Journal on Mathematical Analysis 2012, 44(2): 1175-1210

ISSUE DATE:

2012

URL:

http://hdl.handle.net/2433/158309

RIGHT:

(C) 2012 Society for Industrial and Applied Mathematics 


\title{
INVARIANT MANIFOLDS AROUND SOLITON MANIFOLDS FOR THE NONLINEAR KLEIN-GORDON EQUATION*
}

\author{
K. NAKANISHI ${ }^{\dagger}$ AND W. SCHLAG ${ }^{\ddagger}$
}

\begin{abstract}
We construct center-stable and center-unstable manifolds, as well as stable and unstable manifolds, for the nonlinear Klein-Gordon equation with a focusing energy subcritical nonlinearity, associated with a family of solitary waves which is generated from any radial stationary solution by the action of all Lorentz transforms and spatial translations. The construction is based on the graph transform (or Hadamard) approach, which requires less spectral information on the linearized operator, and less decay of the nonlinearity, than the Lyapunov-Perron method employed previously in this context. The only assumption on the stationary solution is that the kernel of the linearized operator is spanned by its spatial derivatives, which is known to hold for the ground states. The main novelty of this paper is that the graph transform method is carried out in the presence of modulation parameters corresponding to the symmetries.
\end{abstract}

Key words. nonlinear wave equation, nonlinear Klein-Gordon equation, stationary solution, soliton, stable manifold, center-stable manifold

AMS subject classifications. 35L70, 35Q55

DOI. $10.1137 / 11082720 \mathrm{X}$

1. Introduction. Consider the focusing nonlinear Klein-Gordon equation $(\mathrm{NLKG})$ on $\mathbb{R}^{d}$

$$
\ddot{u}-\Delta u+u=f(u), \quad u(t, x): \mathbb{R}^{1+d} \rightarrow \mathbb{R}
$$

where $f: \mathbb{R} \rightarrow \mathbb{R}$ is a given nonlinearity. A typical example is the focusing power nonlinearity

$$
f(u)=|u|^{p-1} u, \quad 3 \leq p+1< \begin{cases}\frac{2 d}{d-2} & (d \geq 3), \\ \infty & (d \leq 2) .\end{cases}
$$

The lower bound can in principle be reduced to $p>1$, but we assume $p \geq 2$ to avoid technical and nonessential complications in the nonlinear estimates.

The equation preserves the total energy and momentum

$$
E(u):=\int_{\mathbb{R}^{d}}\left[\frac{|\dot{u}|^{2}+|\nabla u|^{2}+|u|^{2}}{2}-f^{(-1)}(u)\right] d x, \quad P(u):=\int_{\mathbb{R}^{d}} \dot{u} \nabla u d x,
$$

where $f^{(-1)}: \mathbb{R} \rightarrow \mathbb{R}$ is the primitive $f^{(-1)}(a)=\int_{0}^{a} f(b) d b$. These quantities are well-defined in the energy space

$$
\vec{u}(t):=(u(t), \dot{u}(t)) \in \mathcal{H}:=H^{1}\left(\mathbb{R}^{d}\right) \times L^{2}\left(\mathbb{R}^{d}\right) .
$$

Throughout the paper, we do not distinguish vertical and horizontal vectors in $\mathcal{H}$, except to avoid confusion.

\footnotetext{
* Received by the editors March 11, 2011; accepted for publication (in revised form) December 16, 2011; published electronically April 19, 2012.

http://www.siam.org/journals/sima/44-2/82720.html

${ }^{\dagger}$ Department of Mathematics, Kyoto University, Kyoto 606-8502, Japan (n-kenji@math.kyoto-u. ac.jp).

${ }^{\ddagger}$ Department of Mathematics, University of Chicago, Chicago, IL 60637 (schlag@math.uchicago. edu). This author was supported in part by the National Science Foundation, DMS-0617854.
}

1175 
We will consider NLKG in the energy space $\mathcal{H}$, regarding it as a Hamiltonian system. Our goal is to construct a local center-stable manifold of the family of traveling waves generated by the Lorentz transforms and spatial translations acting on a stationary solution. For brevity, we call the latter manifold of traveling waves the soliton manifold. There are two major approaches used in the construction of centerstable manifolds: the Hadamard method and the Lyapunov-Perron (LP) method. The former uses the evolution backward and locally in time to find a flow-invariant graph of the unstable modes in terms of the other components. (The Hadamard approach also goes by the name of graph transform or invariant cones method.) The latter uses the evolution forward and globally in time to find an initial adjustment of the unstable modes so that they remain small forever.

Bates and Jones [1] developed the Hadamard method in the general setting of an ODE of the form $\dot{x}=A x+f(x)$, where $A$ is an (unbounded) operator on some Banach space $X$ which generates a continuous semigroup, and the nonlinearity $f$ is locally Lipschitz on $X$, satisfies $f(0)=0$, and admits arbitrarily small Lipschitz constants near the equilibrium $x=0$. The spectrum of $A$ is divided in the stable part with eigenvalues in the left half plane, the unstable part which lies in the right half plane, and the center part which lies on the imaginary axis. Assumptions are made on the dimensions of the corresponding spectral subspaces of $X$ and the associated flows (if the spaces are infinite-dimensional) so as to represent two main scenarios: the dissipative case (D) on the one hand and the conservative case (C) on the other hand. For (D) one demands that only the stable subspace be infinite-dimensional and that the associated semigroup is exponentially stable. For (C) only the center subspace is infinite-dimensional, which is precisely what occurs in Hamiltonian problems.

Bates and Jones then verified that the abstract center-stable manifold which they constructed in [1] applies to stationary solutions of NLKG under the radial symmetry restriction for the power nonlinearity (1.2) with $p<\frac{d}{d-2}, d \geq 3$, where the upper bound on $p$ was required to ensure that the nonlinearity is locally Lipschitz $H^{1} \rightarrow L^{2}$. They also showed that if the linearized operator has no nonzero radial functions in its kernel, then

(1) every solution starting on the center-stable manifold stays there forever in positive times, remaining in a small neighborhood of the stationary solution;

(2) every solution starting in that small neighborhood, but off the manifold, must exit the neighborhood in finite positive time.

The kernel condition holds for the ground state (the positive stationary solution) by the work of Weinstein [23].

Gesztesy et al. [14] demonstrated that the Bates-Jones theory applies to the nonlinear Schrödinger equation (NLS) with a spatially localized nonlinearity. Notice that the radial restriction for NLKG prohibits both the spatial translations and the Lorentz transforms, and so the soliton manifold is reduced to a fixed stationary solution. Similarly, the localized nonlinearity of [14] destroys the scaling, translation, and Galilean invariance, so that the soliton can change only with respect to the phase parameter. Indeed, as we will explain below, moving solitons represent a serious obstacle to the Bates-Jones approach.

On the other hand, the second author [22] developed the LP method for the ground state of the cubic NLS in $\mathbb{R}^{3}$ without imposing any symmetry assumptions but in a weighted $H^{s}$-space (or an unweighted $L^{1}$-based space). In this approach, the soliton is allowed to move. Recently, Beceanu [5] extended the latter work to the critical space $\dot{H}^{1 / 2}$ which is bigger than the energy space. Finally, and partly based on a novel approach to linear dispersive estimates developed by Beceanu [4], the authors 
proved in [18] that the LP approach can be carried out for NLKG in the energy space without any symmetry restrictions (see also the book [19]).

However, an essential difficulty in applying the LP method to a nonlinear dispersive equation (without dissipation) is that it requires global dispersive estimates, which in turn necessitate fine spectral information, such as the absence of threshold resonances and of so-called spurious ${ }^{1}$ eigenvalues. Such spectral properties can be very hard to check in higher dimensions, even for the ground state. The onedimensional soliton for monomial powers is explicit, and therefore purely analytical arguments are available; see, for example, [8, 9, 20,21], as well as [15]. For results on the higher-dimensional spectral problem which partially rely on a numerical component, see $[12,13,16]$. For a rigorous proof of the spectral properties of the linearized operators $L_{ \pm}$in radial $\mathbb{R}^{3}$ for the cubic nonlinearity, see [10].

While the LP method requires these stronger spectral ingredients (at least in its current form), it also leads to more detailed conclusions. More specifically, one obtains that solutions starting on the center-stable manifold scatter to the soliton manifold in forward time. In other words, the distinction between the LP approach and the Hadamard approach is roughly tantamount to the distinction between asymptotic and orbital stability of solitary waves.

Alternatively, in the presence of spurious eigenvalues one might hope to invoke the Fermi golden rule which arises in certain delicate normal-form constructions. For a recent application of such ideas to the asymptotic stability problem for a single orbitally stable NLS soliton in the energy space, see [11] and the numerous references there. While such normal form expansions are very natural from the point of view of Hamiltonian stability theory, we do not rely on them here.

In this paper, we employ the Hadamard method in the nonradial setting. Our main challenge is to extend the result by Bates and Jones to the family of traveling waves, rather than stationary ones. We therefore have to investigate the dynamics along the soliton manifold as well, which is usually called the modulational analysis in the stability problem of solitons. In our setting, the soliton manifold has $2 d$ dimensions corresponding to the relativistic momentum and position vectors in $\mathbb{R}^{d}$.

Those parameters can be fixed by means of a Lorentz transform which reduces the total momentum to zero, and by using a coordinate moving with the soliton. In doing so, we encounter a derivative loss due to the translation, i.e., a transport term, in the modulated equation for the difference of two solutions, which disables the contraction argument for the graphs in the energy norm. This difficulty is not an artifact of the coordinate choice but a natural consequence of the two facts that the solitons are translated by the flow, while the translation is not Lipschitz continuous in any Sobolev space. The same problem occurs for any other continuous group action involving a coordinate change, such as scaling or rotation.

We overcome this difficulty by introducing a nonlinear quasi-distance in the energy space, for which the spatial translation becomes Lipschitz continuous, while the topology remains the same. Using the contraction mapping principle with this distance for the continuous spectral part, we are able to carry out the Hadamard method in the presence of modulational parameters.

A more technical issue concerns allowing nonlinearities all the way up to the $H^{1}$ critical power, i.e., for $p<(d+2) /(d-2)$, while Bates and Jones assumed $p<d /(d-2)$. This is easily resolved by using the Strichartz estimate for the free Klein-Gordon equation and by relaxing some flow-invariance conditions by constant multiples.

\footnotetext{
${ }^{1}$ This refers to eigenvalues which do not result from symmetries of the equation.
} 
Since the description of dynamics around the manifolds ((1)-(2) above) is also extended to the current setting, we can easily observe that the maximum backward evolution of the center-stable manifold is identical, in a small neighborhood, to the forward trapping set $\mathcal{T}_{+}$: the collection of initial data for which the solution (of the original NLKG) stays in the small neighborhood for large times.

In the special case where the soliton manifold is generated from the ground state, we can combine the above result with the one-pass theorem in [18] as well as the openness and connectedness of the forward scattering set $\mathcal{S}_{+}$and the forward blowup set $\mathcal{B}_{+}$, thereby concluding that $\mathcal{T}_{+}$separates locally and globally all the solutions with energy at most slightly above the ground state energy into $\mathcal{S}_{+}$and $\mathcal{B}_{+}$. Therefore, the conclusion of [18] is extended to the range

$$
d \in \mathbb{N}, \quad 1+\frac{4}{d}<p<1+\frac{4}{d-2}, \quad p \geq 2,
$$

except for the following scattering statement on $\mathcal{T}_{+}$: all solutions in $\mathcal{T}_{+}$scatter to the soliton manifold as $t \rightarrow \infty$. This statement was proved in [18] for $d=p=3$ by means of the LP method using the following gap property of the linearized operator $L_{+}$:

$(0,1) \cap \sigma\left(L_{+}\right)=\emptyset$, and there is no threshold eigenvalue or resonance.

This is proved (at least for the radial case) in [10]. Note that the numerical analysis of [12] suggests that the absence of threshold resonances and spurious eigenvalues fails for some powers in $(1+4 / 3,3)$ for $d=3$, where the LP method without any hypothesis (typically the Fermi golden rule) is not so far available. Also note that the lower bound $1+4 / d$ is required by the proof of the one-pass theorem but not by the Hadamard construction in this paper, while the LP method also needs it in order to work in the energy space.

To state the main result, we clarify the assumptions on the nonlinearity $f$ and on the stationary solution:

$$
\begin{aligned}
& f \in C^{2}(\mathbb{R} ; \mathbb{R}), \quad 0=f(0)=f^{\prime}(0), \\
& \forall a \in \mathbb{R}, \quad\left|f^{\prime \prime}(a)\right| \lesssim \begin{cases}1+|a|^{p-2} & \left(d \geq 3,2 \leq \exists p<\frac{d+2}{d-2}\right), \\
1+|a|^{p-2} & (d=2,2 \leq \exists p<\infty), \\
\text { arbitrary } & (d=1) .\end{cases}
\end{aligned}
$$

To have $C^{1}$ manifolds, we assume a bit more regularity: for some $p>2$ in the above range,

$$
\left|f^{\prime \prime}\left(a_{1}\right)-f^{\prime \prime}\left(a_{2}\right)\right| \lesssim\left(\left|a_{1}-a_{2}\right|^{p-2}+\left|a_{1}-a_{2}\right|\right)\left[1+\left|a_{1}\right|^{p-3}+\left|a_{2}\right|^{p-3}\right] .
$$

These assumptions are satisfied for example by

$$
f(u)=\sum_{k: \text { finite }} \lambda_{k}|u|^{p_{k}-1} u, \quad \lambda_{k} \geq 0,
$$

provided that all $p_{k}>2$ are in the range (1.6).

Let $Q \in H^{1}\left(\mathbb{R}^{d}\right)$ be a stationary solution of NLKG, i.e., a weak solution of the elliptic PDE

$$
-\Delta Q+Q=f(Q)
$$

Copyright (c) by SIAM. Unauthorized reproduction of this article is prohibited. 
Standard arguments imply that $Q \in H^{2}$ with exponential decay as $|x| \rightarrow \infty$. For existence, see the classical work by Berestycki and Lions [6, 7]. The action of the Lorentz transforms and the spatial translations generate the traveling wave family parametrized by the relativistic momentum $\vec{p} \in \mathbb{R}^{d}$ and position $\vec{q} \in \mathbb{R}^{d}$,

$$
Q(\vec{p}, \vec{q}):=Q\left(x-\vec{q}+\vec{p}(\langle\vec{p}\rangle-1)|\vec{p}|^{-2} \vec{p} \cdot(x-\vec{q})\right),
$$

so that each traveling wave can be written in the form

$$
u(t)=Q(\vec{p}, \vec{q}(t)), \quad \frac{d}{d t} \vec{q}(t)=\frac{\vec{p}(t)}{\langle\vec{p}(t)\rangle} .
$$

For brevity, the spatial translate is denoted also as

$$
Q_{c}(x):=Q(x-c)
$$

The vector form is denoted by

$$
\vec{Q}:=(Q, 0), \quad \vec{Q}(p, q):=\left(Q(p, q),-\frac{\vec{p}}{\langle\vec{p}\rangle} \cdot \nabla Q(p, q)\right),
$$

and the soliton manifold of $Q$ is defined as

$$
\mathscr{S}(Q):=\{\vec{Q}(\vec{p}, \vec{q})\}_{\vec{p}, \vec{q} \in \mathbb{R}^{d}} \subset \mathcal{H},
$$

which is a $C^{1}$ manifold of dimension $2 d$. The linearized operator at $Q$

$$
L_{+}:=D^{2}-f^{\prime}(Q)=-\Delta+1-f^{\prime}(Q), \quad D:=\sqrt{1-\Delta},
$$

is self-adjoint on $L^{2}$ with a finite number of eigenvalues and continuous spectrum $\sigma_{c}\left(L_{+}\right)=\sigma_{a c}\left(L_{+}\right)=[1, \infty)$. The translation invariance of NLKG implies that $L_{+} \nabla Q=0$. The only assumption on $Q$ in this paper is

$$
L_{+}^{-1}(0)=\operatorname{span}\{\nabla Q\} .
$$

This is a well-known property of the ground states. To be more precise, by an argument of Weinstein [23], it holds for the ground state $Q$ provided no radial function lies in the kernel of $L_{+}$. The latter holds for any subcritical monomial nonlinearity (as well as others); see Lemma 2.3 in [17], for example. Moreover, (1.16) seems to be a natural assumption for any other radial static solution. For nonradial static solutions, we have to include angular derivatives as well, but we do not consider such solutions in this paper. Although we will not explicitly use the radial symmetry of $Q$, the reader may assume it without losing anything throughout the paper.

TheOREM 1.1. Let $d \in \mathbb{N}$ and assume that $f$ satisfies (1.6). Let $Q$ be a static solution (1.9) and assume that its linearized operator $L_{+}$satisfies (1.16). Then there is a Lipschitz manifold $\mathcal{M}_{c s}$ in $\mathcal{H}$ containing the soliton manifold $\mathscr{S}(Q)$ with the following properties:

(1) The codimension of $\mathcal{M}_{c s}$ in $\mathcal{H}$ equals the total dimension of the eigenspaces of $L_{+}$corresponding to negative eigenvalues, which we denote by $K$.

(2) $\mathcal{M}_{c s}$ is invariant under the forward evolution of NLKG (1.1).

(3) $\mathcal{M}_{c s}$ is invariant under spatial translations.

Copyright (C) by SIAM. Unauthorized reproduction of this article is prohibited. 
(4) For every Lorentz transform and every $(\vec{p}, \vec{q})$, there is a small neighborhood of $\vec{Q}(\vec{p}, \vec{q})$ such that the Lorentz transform of any forward global solution starting from $\mathcal{M}_{c s}$ within this neighborhood remains on $\mathcal{M}_{c s}$ for all $t>0$.

(5) $\mathcal{M}_{c s}$ is normal at $\vec{Q}$ to the vector $(-k \rho, \rho)$ for any $\rho \in H^{1}$ solving $L_{+} \rho=-k^{2} \rho$ for some $k>0$. In other words,

$$
\left\langle u_{2} \mid \rho\right\rangle=-k\left\langle u_{1}-Q \mid \rho\right\rangle+o\left(\left\|u_{1}-Q\right\|_{H^{1}}+\left\|u_{2}\right\|_{L^{2}}\right) .
$$

(6) For any neighborhood $\mathcal{O}$ of $\mathscr{S}(Q)$, there is a smaller neighborhood $\mathcal{O}^{\prime}$ such that every solution starting from $\mathcal{O}^{\prime} \cap \mathcal{M}_{c s}$ remains in $\mathcal{O} \cap \mathcal{M}_{c s}$ for all $t>0$.

(7) There is a neighborhood $\mathcal{O}$ of $\mathscr{S}(Q)$ such that every solution starting from $\mathcal{O} \backslash \mathcal{M}_{c s}$ exits $\mathcal{O}$ in finite positive time.

(8) If in addition $f$ satisfies (1.7), then $\mathcal{M}_{c s}$ is $C^{1, \alpha}$, where $\alpha=\min (1, p-2)$.

The corresponding statement for a center-unstable manifold follows simply by the time inversion, so we omit it. However, in the proof we will actually consider the center-unstable manifold, for which the forward evolution is used as a contraction mapping in the Hadamard method. The center manifold is obtained by intersecting the center-stable with the center-unstable manifold. It is of codimension $2 K$ and is bi-invariant.

Properties (6) and (7) characterize $\mathcal{M}_{c s}$ as the set of solutions which stay close to $\mathscr{S}(Q)$ for all $t \geq 0$. Since the LP method looks for such solutions from the beginning, it will yield a subset of $\mathcal{M}_{c s}$, and indeed the same manifold (locally), provided that the codimension is the same. An advantage of the LP method is that it implies the scattering to the soliton manifold for the solutions on $\mathcal{M}_{c s}$ (cf. [18]). It will be interesting to see what happens when some spectral condition breaks down, e.g., if there is a threshold resonance.

We also obtain a stable and unstable manifold theorem. Recall the definition of $K$ from the previous theorem.

THEOREM 1.2. Under the assumptions of the previous theorem, there exist Lipschitz manifolds $\mathcal{M}_{u}$ and $\mathcal{M}_{s}$ of dimensions $K+d$ with the following properties:

(1) $\mathcal{M}_{s}$ is invariant under the forward evolution of the NLKG (1.1).

(2) $\mathcal{M}_{s}$ is invariant under spatial translations.

(3) Every solution starting on $\mathcal{M}_{s}$ converges exponentially to $\vec{Q}(\cdot-c(t))$ as $t \rightarrow \infty$ where $\dot{c}(t) \rightarrow 0$ exponentially as $t \rightarrow \infty$.

(4) There exists $\delta>0$ small such that $\mathcal{M}_{s}$ is a Lipschitz graph over $B_{\delta}(0) \times$ $\mathbb{R}^{d}$ with $B_{\delta}(0)$ being a $\delta$-ball in the eigenspace corresponding to the negative eigenvalues and the $\mathbb{R}^{d}$-component deriving from spatial translations.

(5) $\mathcal{M}_{u}$ is obtained from $\mathcal{M}_{s}$ by reversing time.

In closing, let us point out references [3], [2], which contain important work on invariant manifold theory in infinite dimensions which is "abstract" in nature and goes beyond the classical Bates-Jones theorem [1]. It would be most interesting to explore if and to what extent the main theorem on existence of invariant manifolds in [2] is applicable in the setting of this paper.

2. Preliminaries. Here we fix some notation. For any two elements $v^{0}, v^{1}$ in a normed space $V$, the ordered pair and their difference are denoted by

$$
v^{\triangleright}:=\left(v^{0}, v^{1}\right) \in V^{2}, \quad\left\|v^{\triangleright}\right\|_{V}=\left\|v^{0}\right\|_{V}+\left\|v^{1}\right\|_{V}, \quad \triangleleft v^{\triangleright}:=v^{1}-v^{0},
$$

respectively. More generally, for any map $M: V \times W \times \cdots$ and elements $v^{j} \in V$, 
$w^{j} \in W, \ldots$, the mapped pair is denoted by

$$
M\left(v^{\triangleright}, w^{\triangleright}, \ldots\right):=\left(M\left(v^{0}, w^{0}, \ldots\right), M\left(v^{1}, w^{1}, \ldots\right)\right) .
$$

For any $R \in \mathbb{R}$ and $\delta>0$, the minimum is denoted by

$$
(R \wedge \delta):=\min (R, \delta)
$$

As usual, $a \lesssim b, a \gtrsim b$, and $a \simeq b$ involve implicit multiplicative constants.

2.1. Equation and spectrum. The energy space $\mathcal{H}=H^{1} \times L^{2} \subset\left(L^{2}\right)^{2}$ is endowed with the usual inner product

$$
\langle\varphi, \psi\rangle_{\mathcal{H}}:=\int_{\mathbb{R}^{d}}\left[\nabla \varphi_{1} \cdot \nabla \psi_{1}+\varphi_{1} \psi_{1}+\varphi_{2} \psi_{2}\right] d x
$$

and the $L^{2}$ duality coupling

$$
\langle\varphi \mid \psi\rangle:=\int_{\mathbb{R}^{d}}\left[\varphi_{1}(x) \psi_{1}(x)+\varphi_{2}(x) \psi_{2}(x)\right] d x,
$$

as well as the symplectic form

$$
\omega(\varphi, \psi):=\langle J \varphi \mid \psi\rangle=\int_{\mathbb{R}^{d}}\left[\varphi_{2}(x) \psi_{1}(x)-\varphi_{1}(x) \psi_{2}(x)\right] d x,
$$

where $J$ is the skew-symmetric matrix

$$
J:=\left(\begin{array}{cc}
0 & 1 \\
-1 & 0
\end{array}\right), \quad J^{2}=-\left(\begin{array}{cc}
1 & 0 \\
0 & 1
\end{array}\right) .
$$

Let $\mathcal{L}$ be the self-adjoint operator on $L^{2}$ with domain $H^{2} \times L^{2}$

$$
\mathcal{L}:=\left(\begin{array}{cc}
L_{+} & 0 \\
0 & 1
\end{array}\right)=\left(\begin{array}{cc}
-\Delta+1-f^{\prime}(Q) & 0 \\
0 & 1
\end{array}\right) .
$$

Its free version is denoted by

$$
\mathcal{D}:=\left(\begin{array}{cc}
D^{2} & 0 \\
0 & 1
\end{array}\right)=\left(\begin{array}{cc}
-\Delta+1 & 0 \\
0 & 1
\end{array}\right) .
$$

Then the linearized equation around $\vec{Q}=(Q, 0)$ is

$$
v_{t}=J \mathcal{L} v
$$

The spectrum of $J \mathcal{L}$ is given in terms of that of $L_{+}$:

$$
\sigma(J \mathcal{L})= \pm \sqrt{-\sigma\left(L_{+}\right)} .
$$

Since $f^{\prime}(Q)$ is bounded and exponentially decreasing, there are $0<\underline{k} \leq 1<\bar{k}$ and a finite set $K \subset[\underline{k}, \bar{k}]$ such that

$$
\sigma\left(L_{+}\right) \backslash\left[\underline{k}^{2}, \infty\right)=\{0\} \cup\left\{-k^{2} \mid k \in K\right\} .
$$

Copyright (C) by SIAM. Unauthorized reproduction of this article is prohibited. 
With slight abuse of notation, we let $K$ count the multiplicity of each negative eigenvalue $-k^{2}$ as well. For each $k \in K$, let $\rho_{k} \in \mathcal{S}\left(\mathbb{R}^{d}\right)$ be an eigenfunction satisfying

$$
L_{+} \rho_{k}=-k^{2} \rho_{k}, \quad\left\|\rho_{k}\right\|_{2}=1 .
$$

The (generalized) eigenfunctions of $J \mathcal{L}$ are

$$
J \mathcal{L} g_{k \pm}= \pm k g_{k \pm}, \quad J \mathcal{L} \nabla \vec{Q}=0, \quad J \mathcal{L} J \nabla \vec{Q}=-\nabla \vec{Q}, \quad g_{k \pm}:=\left(\begin{array}{c}
1 \\
\pm k
\end{array}\right) \frac{\rho_{k}}{\sqrt{2 k}}
$$

which satisfy

$$
\omega\left(\partial_{\alpha} \vec{Q}, J \partial_{\beta} \vec{Q}\right)=H_{\alpha, \beta}(Q):=\left\langle\partial_{\alpha} Q \mid \partial_{\beta} Q\right\rangle, \quad \omega\left(g_{k+}, g_{k-}\right)=1 .
$$

The corresponding symplectic (spectral) decomposition takes the form

$$
\begin{aligned}
v & =\sum_{ \pm, k \in K} \lambda_{k \pm} g_{k \pm}+\mu \cdot \nabla \vec{Q}+\nu \cdot J \nabla \vec{Q}+\gamma, \\
\lambda_{k \pm} & :=P_{ \pm k} v:=\omega\left(v, \pm g_{k \mp}\right), \\
\mu & :=P_{\mu} v:=H(Q)^{-1} \omega(v, J \nabla \vec{Q}), \quad \nu:=P_{\nu} v:=H(Q)^{-1} \omega(v,-\nabla \vec{Q}), \\
\gamma & :=P_{\gamma} v:=v-\sum_{ \pm, k} \lambda_{ \pm k} g_{k \pm}-\mu \cdot \nabla \vec{Q}-\nu \cdot J \nabla \vec{Q} .
\end{aligned}
$$

We will use as well the projections

$$
\begin{aligned}
v_{ \pm} & :=P_{ \pm} v:=\sum_{k \in K} \lambda_{k \pm} g_{k \pm}, \\
v_{d} & :=P_{d} v:=v-P_{\gamma} v, \\
v_{0} & :=P_{0} v:=\nabla \vec{Q} \cdot \mu+J \nabla \vec{Q} \cdot \nu, \quad v_{\geq 0}:=P_{\geq 0} v:=v-v_{-}, \\
v_{\gamma+} & :=P_{\gamma+} v:=\gamma+v_{+}, \quad v_{0 \pm}:=P_{0 \pm} v:=v_{0}+v_{ \pm}
\end{aligned}
$$

and the corresponding subspaces $\mathcal{H}_{ \pm}:=P_{ \pm}(\mathcal{H})$. Fixing a small number

$$
0<\kappa \ll \underline{k},
$$

we define the energy norm on $\mathcal{H}$ to be

$$
\|v\|_{E}^{2}:=\sum_{k \in K}\left|\lambda_{k}\right|^{2}+|\nu|^{2}+\kappa^{2}|\mu|^{2}+\langle\mathcal{L} \gamma \mid \gamma\rangle \simeq\|v\|_{\mathcal{H}}^{2},
$$

where the final equivalence follows from the orthogonality of $\gamma_{1}$ :

$$
0=\left\langle\gamma_{1} \mid \nabla Q\right\rangle=\left\langle\gamma_{1} \mid \rho_{k}\right\rangle \quad \forall k,
$$

together with (1.16), since $\langle\mathcal{L} \gamma \mid \gamma\rangle=\left\langle L_{+} \gamma_{1} \mid \gamma_{1}\right\rangle+\left\|\gamma_{2}\right\|_{2}^{2}$.

Let $\vec{u} \in C(I ; \mathcal{H})$ be a solution (1.1); then $v=\left(v_{1}, v_{2}\right):=\vec{u}(x+c)-\vec{Q}$ solves

$$
v_{t}=J \mathcal{L} v+\dot{c} \cdot(\nabla \vec{Q}+\nabla v)+\vec{N}(v), \quad \vec{N}(v)=(0, N(v)),
$$

where $N: \mathcal{H} \rightarrow H^{-1}$ carries the superlinear part

$$
N(v):=f\left(Q+v_{1}\right)-f(Q)-f^{\prime}(Q) v_{1}=o\left(v_{1}\right) .
$$

We remark that $v_{2} \neq \dot{v}_{1}$ unless $\dot{c}=0$. 
The conserved momentum can be rewritten as

$$
P(u):=\omega(\vec{u}, \nabla \vec{u}) / 2=\omega(v, \nabla \vec{Q})+\omega(v, \nabla v) / 2 .
$$

By means of a Lorentz transform, we can reduce the dynamics near the soliton manifold $\mathscr{S}(Q)$ to the invariant subspace

$$
\mathcal{H}_{0}:=\{\vec{u} \in \mathcal{H} \mid P(u)=0\} .
$$

Furthermore, we can restrict $v$ to the subspace

$$
\mathcal{H}_{\perp}:=\{v \in \mathcal{H} \mid \omega(v, \nabla \vec{Q})+\omega(v, \nabla v) / 2=0, \omega(v, J \nabla \vec{Q})=0\}
$$

by choosing $\dot{c}$ so that

$$
0=\partial_{t} \omega(v, J \nabla \vec{Q})=\left(H(Q)-\left\langle\nabla^{2} Q \mid v_{1}\right\rangle\right) \dot{c}+\omega(v, \nabla \vec{Q}) .
$$

Hence the evolution for small $v$ on $\mathcal{H}_{\perp}$ is given by

$$
\begin{aligned}
v_{t} & =J \mathcal{L} v+A(v) \cdot \nabla(\vec{Q}+v)+\vec{N}(v), \\
A(v) & :=\left(H(Q)-\left\langle\nabla^{2} Q \mid v_{1}\right\rangle\right)^{-1} \omega(v, \nabla v) / 2 .
\end{aligned}
$$

This is a first-order autonomous equation in $\mathcal{H}$ with the superlinear term

$$
M(v):=A(v) \cdot \nabla(\vec{Q}+v)+\vec{N}(v) .
$$

In order to implement the Hadamard method, we need to localize the nonlinear part $M(v)$ near 0 so that it becomes a small Lipschitz term globally in the energy space $\mathcal{H}$. It seems extremely hard to do this keeping the above orthogonal structure, since the acceleration or the modulation term is naturally unbounded, unless the linearized operator is modified depending on the distance of $v$ from 0 . Therefore we will not enforce the orthogonality conditions but instead solve a localized version of the above autonomous equation in the whole energy space $\mathcal{H}$. After constructing a center-unstable manifold by the Hadamard method for the localized equation, we will restrict that manifold to the subspace $\mathcal{H}_{\perp}$ in a small neighborhood of 0 to obtain a center-unstable manifold for the true equation.

In the case of the unstable manifold, the exponential decay of $v$ as $t \rightarrow-\infty$ ensures that the manifold for the localized equation around 0 falls into $\mathcal{H}_{\perp}$, so that we can automatically get the manifold of the true equation.

2.2. Mobile distance. The most serious obstacle to carrying out the graph transform method in the nonradial setting results from the contraction step in the construction of the invariant graphs, where the presence of the unbounded translation term causes problems. To remedy this, we introduce the mobile distance on $\mathcal{H}$. Heuristically speaking, the standard $L^{p}$ or Sobolev-type norm is too tight for "horizontal motion" $\varphi \mapsto \varphi\left(\cdot+x_{0}\right)$ compared with "vertical motion" $\varphi \mapsto \lambda \varphi$. The mobile distance makes translation just as easy as amplification, without changing the topology.

DeFinition 2.1. For any continuous increasing function $\phi:[0, \infty) \rightarrow[0, \infty)$ satisfying

(1) $\phi(a) \geq a$,

(2) $a \leq 2 b \Longrightarrow \phi(b) \leq 4 \phi(a)$, 
the mobile distance $\mathfrak{m}_{\phi}: \mathcal{H} \times \mathcal{H} \rightarrow[0, \infty)$ is defined by

$$
\mathfrak{m}_{\phi}\left(v^{0}, v^{1}\right)^{2}:=\inf _{q \in \mathbb{R}^{d}, j=0,1}\left\|v^{1-j}-v^{j}(\cdot-q)\right\|_{E}^{2}+|q|^{2} \phi\left(\left\|v^{j}\right\|_{E}\right)^{2} .
$$

Obviously, the infimum in (2.28) is attained at some $q \in \mathbb{R}^{d} \cdot \mathfrak{m}_{\phi}$ is not really a distance but is a quasi-distance on $\mathcal{H}$. More precisely, we have the following proposition.

Proposition 2.2. $\mathfrak{m}_{\phi}$ in (2.28) is a complete quasi-distance on $\mathcal{H}$, satisfying

(1) $\mathfrak{m}_{\phi}\left(v^{0}, v^{1}\right) \geq 0$, where the equality holds iff $v^{0}=v^{1}$,

(2) $\mathfrak{m}_{\phi}\left(v^{1}, v^{0}\right)=\mathfrak{m}_{\phi}\left(v^{0}, v^{1}\right)$,

(3) $\mathfrak{m}_{\phi}\left(v^{0}, v^{1}\right) \leq C_{d}\left[\mathfrak{m}_{\phi}\left(v^{0}, v^{2}\right)+\mathfrak{m}_{\phi}\left(v^{2}, v^{1}\right)\right]$ for some absolute constant $C_{d}>0$,

(4) if $\mathfrak{m}_{\phi}\left(v^{m}, v^{n}\right) \rightarrow 0(n, m \rightarrow \infty)$, then $v^{n}$ converges in $\mathcal{H}$.

Moreover, it satisfies with some absolute constant $C>0$,

$$
\left|\left\|v^{0}\right\|_{\mathcal{H}}-\left\|v^{1}\right\|_{\mathcal{H}}\right|+\left\|D^{-1}\left(v^{0}-v^{1}\right)\right\|_{\mathcal{H}} \leq C \mathfrak{m}_{\phi}\left(v^{0}, v^{1}\right) \leq C^{2}\left\|v^{0}-v^{1}\right\|_{\mathcal{H}},
$$

where $D:=\sqrt{1-\Delta}$. These constants, $C$ and $C_{d}$, do not depend on the choice of $\phi$.

Hence $\mathfrak{m}_{\phi}$ defines the same topology as $\mathcal{H}$, differing only in terms of uniformity. For example, for any $\varphi \in \mathcal{H}$ we have

$$
\lim _{n \rightarrow \infty} \mathfrak{m}_{\phi}\left(\varphi e^{i n x_{1}},-\varphi e^{i n x_{1}}\right)=O(1),
$$

since $-\varphi e^{i n x_{1}}=\varphi e^{i n\left(x_{1}+\pi / n\right)}$, whereas $\left\|\varphi e^{i n x_{1}}-\left(-\varphi e^{i n x_{1}}\right)\right\|_{\mathcal{H}}=O(n)$ unless $\varphi=0$.

Proof of Proposition 2.2. Items (1) and (2) are obvious. For the leftmost term of (2.29), and with $\tau_{q} v:=v(\cdot-q)$,

$$
\begin{aligned}
\left|\left\|v^{0}\right\|_{\mathcal{H}}-\left\|v^{1}\right\|_{\mathcal{H}}\right| & \leq \inf _{q} \min \left(\left\|v^{0}-\tau_{q} v^{1}\right\|_{\mathcal{H}},\left\|v^{1}-\tau_{q} v^{0}\right\|_{\mathcal{H}}\right) \\
& \lesssim \mathfrak{m}_{\phi}\left(v^{0}, v^{1}\right)
\end{aligned}
$$

for the second term,

$$
\begin{aligned}
\left\|D^{-1}\left(v^{0}-v^{1}\right)\right\|_{\mathcal{H}} & \leq\left\|D^{-1}\left(v^{0}-\tau_{q} v^{1}\right)\right\|_{\mathcal{H}}+\left\|D^{-1}\left(\tau_{q} v^{1}-v^{1}\right)\right\|_{\mathcal{H}} \\
& \lesssim\left\|v^{0}-\tau_{q} v^{1}\right\|_{\mathcal{H}}+|q|\left\|\nabla D^{-1} v^{1}\right\|_{\mathcal{H}} \\
& \lesssim\left\|v^{0}-\tau_{q} v^{1}\right\|_{E}+|q| \phi\left(\left\|v^{1}\right\|_{E}\right),
\end{aligned}
$$

while the right bound in (2.29) is obvious by choosing $q=0$. Next we prove the quasi-triangle inequality. For any $v^{0}, v^{1}, v^{2} \in \mathcal{H}$, there are $q^{1}, q^{2} \in \mathbb{R}^{d}$ such that

$$
\mathfrak{m}_{\phi}\left(v^{0}, v^{j}\right) \simeq\left\|v^{0}-\tau_{q^{j}} v^{j}\right\|_{\mathcal{H}}+\left|q^{j}\right| \phi\left(\min \left(\left\|v^{0}\right\|_{\mathcal{H}},\left\|v^{j}\right\|_{\mathcal{H}}\right)\right) \quad(j=1,2)
$$

since the $\mathcal{H}$ and $E$ norms are equivalent and the $\mathcal{H}$ norm is translation invariant. If $\left\|v^{0}\right\|_{\mathcal{H}} \ll \min \left(\left\|v^{1}\right\|_{\mathcal{H}},\left\|v^{2}\right\|_{\mathcal{H}}\right)$, then $\mathfrak{m}_{\phi}\left(v^{0}, v^{j}\right) \simeq\left\|v^{j}\right\|_{\mathcal{H}}$, and so the quasi-triangle inequality is obvious. Otherwise,

$$
\begin{aligned}
\mathfrak{m}_{\phi}\left(v^{1}, v^{2}\right) & \lesssim\left\|v^{1}-v^{2}\left(\cdot-q^{2}+q^{1}\right)\right\|_{\mathcal{H}}+\left|q^{1}-q^{2}\right| \phi\left(\min \left(\left\|v^{1}\right\|_{\mathcal{H}},\left\|v^{2}\right\|_{\mathcal{H}}\right)\right) \\
& \lesssim\left\|v^{1}\left(\cdot-q^{1}\right)-v^{2}\left(\cdot-q^{2}\right)\right\|_{\mathcal{H}}+\sum_{j=1,2}\left|q^{j}\right| \phi\left(\min \left(\left\|v^{0}\right\|_{\mathcal{H}},\left\|v^{j}\right\|_{\mathcal{H}}\right)\right) \\
& \lesssim \mathfrak{m}_{\phi}\left(v^{0}, v^{1}\right)+\mathfrak{m}_{\phi}\left(v^{0}, v^{2}\right) .
\end{aligned}
$$

Copyright (c) by SIAM. Unauthorized reproduction of this article is prohibited. 
To prove the completeness, let $v^{n}$ be Cauchy in $\mathfrak{m}_{\phi}$. Then so is $D^{-1} v^{n}$ in $\mathcal{H}$ by (2.29). Hence, $v^{n}$ converges to some $v \in D \mathcal{H}$. Equation (2.29) implies that $\left\|v^{n}\right\|_{\mathcal{H}}$ converges. We may assume that this limit is positive, since otherwise the convergence to 0 is obvious. Since $v^{n}$ is bounded in $\mathcal{H}$, it converges weakly to $v$ in $\mathcal{H}$. Passing to a subsequence, we may find $q_{n} \in \mathbb{R}^{d}$ such that

$$
\left\|\tau_{q_{n}} v^{n}-v^{n+1}\right\|_{\mathcal{H}}+\left|q_{n}\right|<2^{-n} .
$$

Let $c_{n}=\sum_{k \geq n} q_{n}$; then $c_{n} \rightarrow 0$ and

$$
\left\|v^{n}\left(\cdot-c_{n}\right)-v^{n+1}\left(\cdot-c_{n+1}\right)\right\|_{\mathcal{H}}<2^{-n}
$$

which implies $\tau_{c_{n}} v^{n} \rightarrow v$ strongly in $\mathcal{H}$, whence also $v^{n} \rightarrow v$ strongly in $\mathcal{H}$.

We apply the mobile distance only to the continuous spectrum part because on the one hand, the discrete spectral part is finite-dimensional and smooth, and on the other hand, the linearized energy is conserved only on the continuous spectrum. Choose positive constants $\delta, C_{0}, C_{1}, C_{2}$ such that

$$
0<C_{2} \delta \ll 1 \ll C_{0} \ll C_{1} \ll C_{2} .
$$

The required smallness of $C_{2} \delta, 1 / C_{0}, C_{0} / C_{1}$, and $C_{1} / C_{2}$ is implicit in the following arguments, but only in terms of $d, f, Q$, and $\kappa$. Henceforth, we shall regard those $C_{j}$ as being fixed constants and ignore the dependence on them unless it is important, while we regard $\delta$ as a small parameter (with the smallness depending on $C_{2}$ ), keeping track of its impact on the estimates.

The quasi-distance $\tilde{\mathfrak{m}}_{\delta}: \mathcal{H} \times \mathcal{H} \rightarrow[0, \infty)$ is defined by

$$
\tilde{\mathfrak{m}}_{\delta}\left(v^{0}, v^{1}\right)^{2}:=\left\|P_{d}\left(v^{0}-v^{1}\right)\right\|_{E}^{2}+\mathfrak{m}_{\phi_{\delta}}\left(P_{\gamma} v^{0}, P_{\gamma} v^{1}\right)^{2},
$$

where $\phi_{\delta}(a):=\phi(a / \delta)$ with a fixed $\phi \in C^{\infty}(\mathbb{R})$ satisfying

$$
\phi(a)=\left\{\begin{array}{ll}
1 & \left(a \leq C_{2}\right), \\
a & \left(a \geq 2 C_{2}\right),
\end{array} \quad 0 \leq \phi^{\prime} \leq 1 .\right.
$$

We will localize the equation for $v$ within distance $O(\delta)$ from 0 such that the evolution outside of it becomes purely linearized. We have chosen $\phi_{\delta}$ such that the "fare" is purely proportional to the translation distance within the nonlinear region, but there is an additional fee for "excessive weight" over $O(\delta)$. It is easy to see that $\tilde{\mathfrak{m}}_{\delta}$ has the same properties as $\mathfrak{m}_{\phi}$ in Proposition 2.2.

3. Construction of manifolds for a localized equation. In this section we construct a global center-unstable manifold for an equation of $v$ with localized nonlinearity around $0 \in \mathcal{H}$. The manifold obeys the original flow only on the subset $\mathcal{H}_{\perp}$ in a small neighborhood.

3.1. Localization of the equation. Let $\chi \in C_{0}^{\infty}(\mathbb{R})$ be a nonnegative symmetric decreasing function satisfying $\chi(t)=1$ for $|t| \leq 1$ and $\chi(t)=0$ for $|t| \geq 2$. Let

$$
\chi_{\delta}(v):=\chi\left(\|v\|_{\mathcal{H}}^{2} / \delta^{2}\right)
$$


We will construct a center-unstable manifold near 0 for the equation of $v$ with the nonlinearity localized within $O(\delta)$ distance from 0

$$
v_{t}=J \mathcal{L} v+M_{\delta}(v), \quad M_{\delta}(v):=\chi_{\delta}(v)[A(v) \cdot \nabla(\vec{Q}+v)+\vec{N}(v)] .
$$

Hence each component in the spectral decomposition solves

$$
\begin{aligned}
\partial_{t} \lambda_{k \pm} & = \pm k \lambda_{k \pm}+P_{ \pm k} M_{\delta}(v), \\
\partial_{t} \mu & =-\nu+P_{\mu} M_{\delta}(v), \quad \partial_{t} \nu=P_{\nu} M_{\delta}(v), \\
\partial_{t} \gamma & =J \mathcal{L} \gamma+P_{\gamma} M_{\delta}(v) .
\end{aligned}
$$

Lemma 3.1. The equation (3.2) is globally well-posed in $\mathcal{H}$, and for any solution $v$,

$$
\begin{aligned}
\sup _{|t| \leq 1}\|v(t)\|_{E} & \lesssim\|v(0)\|_{E}, \\
\sup _{|t| \leq 1}\left\|v_{d}(t)-e^{J \mathcal{L} t} v_{d}(0)\right\|_{E} & \lesssim\left(\|v(0)\|_{E} \wedge \delta\right)^{2}, \\
\sup _{|t| \leq 1}\left|\|\gamma(t)\|_{E}^{2}-\|\gamma(0)\|_{E}^{2}\right| & \lesssim\left(\|v(0)\|_{E} \wedge \delta\right)^{3} .
\end{aligned}
$$

Proof. Let $v$ be a local solution around $t=0$, and let $w(t, x)=v(t, x-c)$, where $c$ is the solution of

$$
\dot{c}=\chi_{\delta}(v) A(v), \quad c(0)=0 .
$$

Let $\tau_{c}$ be the translation operator

$$
\tau_{c} \varphi(x)=\varphi(x-c)
$$

then the equation for $(w, c)$ is given by

$$
\dot{w}=J \mathcal{D} w+F(w, c), \quad \dot{c}=B(w, c),
$$

with the nonlinear terms $F$ and $B$ defined by

$$
B(w, c):=\chi_{\delta}(w) A_{c}(w), \quad F(w, c):=\left(\begin{array}{c}
B(w, c) \cdot \nabla Q_{c} \\
f^{\prime}\left(Q_{c}\right) w_{1}+\chi_{\delta}(w) N_{c}(w)
\end{array}\right),
$$

where $A_{c}, N_{c}$ are translates of $A, N$ :

$$
\begin{aligned}
& A_{c}(w):=A\left(\tau_{c}^{*} w\right)=\left(H(Q)-\left\langle\nabla Q_{c} \mid w_{1}\right\rangle\right)^{-1} \omega(w, \nabla w) / 2, \\
& N_{c}(w):=\tau_{c} N\left(\tau_{c}^{*} w\right)=f\left(Q_{c}+w_{1}\right)-f\left(Q_{c}\right)-f^{\prime}\left(Q_{c}\right) w_{1} .
\end{aligned}
$$

Choosing some appropriate Strichartz norm, for example,

$$
\|w\|_{\mathrm{Str}}:= \begin{cases}\|w\|_{L_{t}^{\infty} \mathcal{H}_{x}}+\left\|w_{1}\right\|_{L_{t}^{p} L_{x}^{2 p}} & (d \geq 3) \\ \|w\|_{L_{t}^{\infty} \mathcal{H}_{x}} & (d \leq 2)\end{cases}
$$

with $p=\frac{d+2}{d-2}$, we have by the Strichartz estimate for the free Klein-Gordon equation

$$
\left\|w_{1}\right\|_{\operatorname{Str}(0, T)} \lesssim\|w(0)\|_{\mathcal{H}}+\|F\|_{L_{t}^{1} \mathcal{H}(0, T)}, \quad\|c\|_{L^{\infty}(0, T)} \lesssim|c(0)|+T\|B\|_{L^{\infty}(0, T)},
$$

Copyright $@$ by SIAM. Unauthorized reproduction of this article is prohibited. 
where the nonlinear terms are estimated by Hölder,

$$
\begin{gathered}
F(0, c)=0, \quad B(0, c)=0, \\
\left|\triangleleft B\left(w^{\triangleright}, c^{\triangleright}\right)\right| \lesssim\left[\left|\triangleleft c^{\triangleright}\right|\left(\left\|w^{\triangleright}\right\|_{\mathcal{H}} \wedge \delta\right)^{2}+\left(\left\|\triangleleft w^{\triangleright}\right\|_{\mathcal{H}} \wedge \delta\right)\right]\left(\left\|w^{\triangleright}\right\|_{\mathcal{H}} \wedge \delta\right), \\
\left\|\triangleleft F\left(w^{\triangleright}, c^{\triangleright}\right)\right\|_{L_{t}^{1} \mathcal{H}(0, T)} \lesssim T\left[\left\|\triangleleft c^{\triangleright}\right\|_{L^{\infty}}\left\|w^{\triangleright}\right\|_{\mathrm{Str}}+\left\|\triangleleft w^{\triangleright}\right\|_{\text {Str }}\right] \\
+\left[\left\|\triangleleft c^{\triangleright}\right\|_{L^{\infty}}+\left\|\triangleleft w^{\triangleright}\right\|_{\mathrm{Str}}\right]\left(\left\|w^{\triangleright}\right\|_{\operatorname{Str}} \wedge \delta\right)
\end{gathered}
$$

on the time interval $0<t<T \ll 1$. Hence if $\delta, T>0$ are small enough, we obtain a local solution of $(w, c)$ on $(0, T)$ in $\mathcal{H} \times \mathbb{R}^{d}$ by the standard iteration. By Gronwall, it is extended to any finite time intervals. In particular,

$$
\left\|w_{1}\right\|_{\operatorname{Str}(-1,1)} \lesssim\|w(0)\|_{\mathcal{H}}, \quad|c|_{L^{\infty}(-1,1)} \lesssim|c(0)|+\left(\|w(0)\|_{\mathcal{H}} \wedge \delta\right)^{2},
$$

and moreover, if $\|w(0)\|_{\mathcal{H}} \gg \delta$, then $\|w(t)\|_{\mathcal{H}} \gg \delta$ for $|t| \leq 1$, and so $c(t)=0$ and $F=\left(0, f^{\prime}(Q) w_{1}\right)$. Hence we obtain by the usual iteration and continuation argument

$$
\begin{aligned}
\left\|\triangleleft w^{\triangleright}\right\|_{\operatorname{Str}(-1,1)} & \lesssim\left\|\triangleleft w^{\triangleright}(0)\right\|_{\mathcal{H}}, \\
\left\|\triangleleft c^{\triangleright}\right\|_{L^{\infty}(-1,1)} & \lesssim\left(\left\|\triangleleft w^{\triangleright}(0)\right\|_{\mathcal{H}} \wedge \delta\right)\left(\left\|w^{\triangleright}(0)\right\|_{\mathcal{H}} \wedge \delta\right), \\
\left\|\triangleleft F\left(w^{\triangleright}, c^{\triangleright}\right)\right\|_{L_{t}^{1} \mathcal{H}_{x}(-1,1)} & \lesssim\left\|\triangleleft w^{\triangleright}(0)\right\|_{\mathcal{H}} .
\end{aligned}
$$

Next we prove the second and third estimates in (3.4). Since they are now obvious for $\|v(0)\|_{\mathcal{H}} \geq C_{0} \delta$, we may assume that $\|v(0)\|_{\mathcal{H}} \leq C_{0} \delta$. For the $v_{d}$ part, we have by the energy inequality

$$
\left\|v_{d}-e^{J \mathcal{L} t} v_{d}(0)\right\|_{L_{t}^{\infty} E(0, T)} \leq\left\|D^{-2} M_{\delta}(v)\right\|_{L_{t}^{1} \mathcal{H}_{x}(0, T)} \lesssim\|v\|_{\operatorname{Str}(0, T)}^{2} \lesssim\|v(0)\|_{\mathcal{H}}^{2} .
$$

For the $\gamma$ part, we have

$$
\partial_{t}\langle\mathcal{L} \gamma \mid \gamma\rangle=\chi_{\delta}(v)\left[A(v)\left\langle f(Q) \gamma_{1} \mid \nabla \gamma_{1}\right\rangle+A(v)\left\langle\mathcal{L} \nabla v_{d} \mid \gamma\right\rangle+\left\langle N(v) \mid \gamma_{2}\right\rangle\right],
$$

and so

$$
\begin{aligned}
{\left[\|\gamma\|_{E}^{2}\right]_{0}^{T} } & \lesssim\left\|\left(A(v) f^{\prime}(Q) \gamma_{1}, A(v) D^{2} v_{d}, N(v)\right)\right\|_{L_{t}^{1} L_{x}^{2}(0, T)}\|\gamma\|_{L_{t}^{\infty} \mathcal{H}(0, T)} \\
& \lesssim\|v(0)\|_{\mathcal{H}}^{3}
\end{aligned}
$$

and we are done.

Denote the nonlinear propagator for $(3.2)$ on $\mathcal{H}$ by

$$
U(t): \mathcal{H} \rightarrow \mathcal{H}, \quad U(t) v(0)=v(t) .
$$

3.2. Smallness of nonlinearity. The following estimate on the nonlinear term by the mobile distance will be the basis of all the succeeding arguments.

LEMMA 3.2. For any two solutions $v^{j}(t)=U(t) v^{j}(0) \in C(\mathbb{R} ; \mathcal{H})$ of $(3.2)$, we have

$$
\begin{array}{r}
\sup _{|t| \leq 1} \tilde{\mathfrak{m}}_{\delta} v^{\triangleright}(t) \lesssim \tilde{\mathfrak{m}}_{\delta} v^{\triangleright}(0), \\
\sup _{|t| \leq 1}\left\|P_{d}\left[\triangleleft v^{\triangleright}(t)-e^{J \mathcal{L} t} \triangleleft v^{\triangleright}(0)\right]\right\|_{E}+\left|\left[\tilde{\mathfrak{m}}_{\delta} P_{\gamma} v^{\triangleright}\right]_{0}^{t}\right| \lesssim \delta \tilde{\mathfrak{m}}_{\delta} v^{\triangleright}(0),
\end{array}
$$

where the implicit constants are determined by $d, f, Q$, and $\kappa$.

Copyright (c) by SIAM. Unauthorized reproduction of this article is prohibited. 
1188

Proof. Without loss of generality, we may assume that for some $q(t) \in \mathbb{R}^{d}$

$$
\tilde{\mathfrak{m}}_{\delta} v^{\triangleright}(t)^{2}=\left\|P_{d} \triangleleft v^{\triangleright}(t)\right\|_{E}^{2}+\left\|\gamma^{0}(t)-\tau_{q(t)} \gamma^{1}(t)\right\|_{E}^{2}+|q(t)|^{2} \phi_{\delta}\left(\left\|\gamma^{1}(t)\right\|_{E}\right)^{2}
$$

for $|t| \leq 1$. Decompose each solution by

$$
v^{j}=\sum_{ \pm, k \in K} \lambda_{k \pm}^{j} g_{k \pm}+\mu^{j} \cdot \nabla \vec{Q}+\nu^{j} \cdot J \nabla \vec{Q}+\gamma^{j} .
$$

Case (I): $\left\|\boldsymbol{v}^{\triangleright}(\mathbf{0})\right\|_{\mathcal{H}} \leq \boldsymbol{C}_{\mathbf{1}} \boldsymbol{\delta}$. The previous lemma implies that $\left\|v^{\triangleright}(t)\right\|_{E} \ll C_{2} \delta$, and so $\phi_{\delta}\left(\left\|\gamma^{j}(t)\right\|_{E}\right)=1$, for $|t| \leq 1$ and $j=0,1$. The discrete components solve

$$
\begin{aligned}
\partial_{t} \triangleleft \lambda_{k \pm}^{\triangleright} & = \pm k \triangleleft \lambda_{k \pm}^{\triangleright}+P_{ \pm k} \triangleleft M_{\delta}\left(v^{\triangleright}\right), \\
\partial_{t} \triangleleft \mu^{\triangleright} & =-\triangleleft \nu^{\triangleright}+P_{\mu} \triangleleft M_{\delta}\left(v^{\triangleright}\right), \quad \partial_{t} \triangleleft \nu^{\triangleright}=P_{\nu} \triangleleft M_{\delta}\left(v^{\triangleright}\right),
\end{aligned}
$$

where the nonlinear term is bounded by

$$
\left\|\triangleleft M_{\delta}\left(v^{\triangleright}\right)\right\|_{H^{-2}} \lesssim \delta \tilde{\mathfrak{m}}_{\delta} v^{\triangleright}
$$

which is proved as follows: (2.29) and the translation invariance of $\omega(v, \nabla v)$ imply

$$
\begin{aligned}
\left|\triangleleft \chi_{\delta}^{\triangleright}(v)\right| & \lesssim\left|\triangleleft\left\|v^{\triangleright}\right\|_{2}\right| / \delta \lesssim \tilde{\mathfrak{m}}_{\delta} v^{\triangleright} / \delta, \\
\left|\triangleleft A\left(v^{\triangleright}\right)\right| & \lesssim \delta\left[\left\|D^{-1} \triangleleft v^{\triangleright}\right\|_{\mathcal{H}}+\left\|\gamma^{0}-\tau_{q} \gamma^{1}\right\|_{\mathcal{H}}\right] \lesssim \delta \tilde{\mathfrak{m}}_{\delta} v^{\triangleright}, \\
\left\|\triangleleft \nabla v^{\triangleright}\right\|_{H^{-2}} & \lesssim\left\|D^{-1} \triangleleft v^{\triangleright}\right\|_{\mathcal{H}} \lesssim \tilde{\mathfrak{m}}_{\delta} v^{\triangleright} .
\end{aligned}
$$

The nonlinear part is estimated by using Sobolev

$$
\begin{aligned}
\left\|\triangleleft N\left(v^{\triangleright}\right)\right\|_{H^{-2}} & \lesssim\left\|N\left(v^{0}\right)-\tau_{q} N\left(v^{1}\right)\right\|_{L^{\varrho}}+\int_{0}^{1}\left\|\partial_{\theta} \tau_{\theta q} N\left(v^{1}\right)\right\|_{H_{\varrho}^{-1}} d \theta \\
& \lesssim \delta\left\|v_{1}^{0}-\tau_{q} v_{1}^{1}\right\|_{H^{1}}+\mid q\|\| v_{1}^{1} \|_{H^{1}}^{2} \lesssim \delta \tilde{\mathfrak{m}}_{\delta} v^{\triangleright}
\end{aligned}
$$

where $\varrho:=\min (2,1+1 / p)$ for $d \geq 2$ and $\varrho:=1$ for $d=1$. Thus we obtain (3.23).

Therefore, we have for $|t| \leq 1$,

$$
\left\|\triangleleft v_{d}^{\triangleright}(t)-e^{J \mathcal{L} t} \triangleleft v_{d}^{\triangleright}(0)\right\|_{E} \lesssim\left|\int_{0}^{t} \delta \tilde{\mathfrak{m}}_{\delta} v^{\triangleright}(s) d s\right| .
$$

The linearized solution enjoys the obvious bound

$$
\left\|e^{J \mathcal{L} t} \triangleleft v_{d}^{\triangleright}(0)\right\|_{E} \lesssim e^{\bar{k} t}\left\|\triangleleft v_{d}^{\triangleright}(0)\right\|_{E} .
$$

For the difference in the $\gamma$ component, we need the mobile distance. Let

$$
\tau^{j}:=\tau_{c^{j}}, \quad \zeta^{j}:=\tau^{j} \gamma^{j}, \quad Q^{j}:=\tau^{j} Q=Q\left(\cdot-c^{j}\right),
$$

for $j=0,1$, where $c^{j}(t) \in \mathbb{R}^{d}$ are the solutions of

$$
\dot{c}^{j}=\chi_{\delta}\left(v^{j}\right) A\left(v^{j}\right), \quad c^{0}(0)=0, \quad c^{1}(0)=q(0),
$$

where $q$ has been chosen in (3.20). Then we have

$$
\dot{\zeta}^{j}=J \mathcal{L}^{j} \zeta^{j}+\dot{c}^{j} \cdot\left(P_{\gamma}^{j} \nabla P_{d}^{j} \zeta^{j}-P_{d}^{j} \nabla \zeta^{j}\right)+P_{\gamma}^{j}\left(0, M^{j}\right),
$$

Copyright (c) by SIAM. Unauthorized reproduction of this article is prohibited. 
where $\mathcal{L}^{j}, P_{d}^{j}$, and $P_{\gamma}^{j}$ are linear operators and $M^{j}$ is the nonlinear part, defined by

$$
\mathcal{L}^{j}:=\tau^{j} \mathcal{L}\left(\tau^{j}\right)^{*}, \quad P_{\star}^{j}:=\tau^{j} P_{\star}\left(\tau^{j}\right)^{*}, \quad M^{j}:=\chi_{\delta}\left(v^{j}\right) N_{c^{j}}\left(z^{j}\right),
$$

and $z^{j}:=\tau^{j} v_{d}^{j}+\zeta^{j}$. Hence the difference satisfies

$$
\triangleleft \dot{\zeta}=J \mathcal{L}^{0} \triangleleft \zeta^{\triangleright}+P_{\gamma}^{0}\left(0, \triangleleft M^{\triangleright}\right)+R, \quad\|R\|_{\mathcal{H}} \lesssim \delta\left[\tilde{\mathfrak{m}}_{\delta} v^{\triangleright}+\left|\triangleleft c^{\triangleright}\right|+\left\|\triangleleft \zeta^{\triangleright}\right\|_{2}\right],
$$

using (3.24) as well as $\left\|v^{j}\right\|_{\mathcal{H}} \lesssim \delta$. The Strichartz estimate for the free Klein-Gordon equation yields (regarding $J\left(\mathcal{L}^{0}-\mathcal{D}\right) \triangleleft \zeta^{\triangleright}$ as a perturbation, which can be done by partitioning the time interval)

$$
\left\|\triangleleft \zeta^{\triangleright}\right\|_{\operatorname{Str}(0,1)} \lesssim\left\|\triangleleft \zeta^{\triangleright}(0)\right\|_{2}+\left\|\triangleleft M^{\triangleright}\right\|_{L_{t}^{1} L_{x}^{2}(0,1)}+\|R\|_{L_{t}^{\infty} \mathcal{H}_{x}(0,1)},
$$

where the nonlinear part is estimated by applying Hölder to the second-order Taylor expansion of $f$,

$$
f\left(Q^{j}+z_{1}^{j}\right)-f\left(Q^{j}\right)-f^{\prime}\left(Q^{j}\right) z_{1}^{j}=\int_{0}^{1} \int_{0}^{1}\left[f^{\prime \prime}\left(Q^{j}+\alpha \theta z_{1}^{j}\right) \theta\left(z_{1}^{j}\right)^{2}\right] d \alpha d \theta,
$$

and

$$
\begin{aligned}
& \triangleleft\left[f\left(Q^{\triangleright}+z_{1}^{\triangleright}\right)-f\left(Q^{\triangleright}\right)-f^{\prime}\left(Q^{\triangleright}\right) z_{1}^{\triangleright}\right] \\
& \quad=\int_{0}^{1} \int_{0}^{1}\left[f^{\prime \prime}\left(Q^{\alpha}+\theta z_{1}^{\alpha}\right) \triangleleft\left(Q^{\triangleright}+\theta z_{1}^{\triangleright}\right)+f^{\prime \prime}\left(Q^{0}+\alpha \theta z_{1}^{0}\right) \theta z_{1}^{0} \triangleleft z_{1}^{\triangleright}\right] d \alpha d \theta,
\end{aligned}
$$

where $Q^{\alpha}:=(1-\alpha) Q^{0}+\alpha Q^{1}$ and $z^{\alpha}:=(1-\alpha) z^{0}+\alpha z^{1}$. Thus we get

$$
\left\|\triangleleft M^{\triangleright}\right\|_{L_{t}^{1} L_{x}^{2}(0,1)} \lesssim\left(1+\left\|z^{\triangleright}\right\|_{\operatorname{Str}(0,1)}\right)^{p-2}\left\|z^{\triangleright}\right\|_{\operatorname{Str}(0,1)}\left[\left\|\triangleleft c^{\triangleright}\right\|_{L_{t}^{\infty}(0,1)}+\left\|\triangleleft z^{\triangleright}\right\|_{\operatorname{Str}(0,1)}\right] .
$$

On the other hand, we have

$$
\begin{aligned}
\left\|\triangleleft z^{\triangleright}\right\|_{\operatorname{Str}(0,1)} & \lesssim\left\|\triangleleft c^{\triangleright}\right\|_{L^{\infty}(0,1)}+\left\|\triangleleft v_{d}^{\triangleright}\right\|_{L^{\infty} \mathcal{H}(0,1)}+\left\|\triangleleft \zeta^{\triangleright}\right\|_{\operatorname{Str}(0,1)}, \\
\tilde{\mathfrak{m}}_{\delta} v^{\triangleright} & \lesssim\left\|\triangleleft v_{d}^{\triangleright}\right\|_{\mathcal{H}}+\left\|\triangleleft \zeta^{\triangleright}\right\|_{\mathcal{H}}+\left|\triangleleft c^{\triangleright}\right| .
\end{aligned}
$$

Combining these estimates with (3.33)-(3.36), (3.26), and (3.27), we obtain

$$
\sup _{0 \leq t \leq 1} \tilde{\mathfrak{m}}_{\delta} v^{\triangleright} \lesssim\left\|\triangleleft c^{\triangleright}\right\|_{C_{t}^{1}(0,1)}+\left\|\triangleleft \zeta^{\triangleright}\right\|_{\operatorname{Str}(0,1)}+\left\|\triangleleft v_{d}^{\triangleright}\right\|_{\operatorname{Str}(0,1)} \lesssim \tilde{\mathfrak{m}}_{\delta} v^{\triangleright}(0) .
$$

For the sharper estimate on the $\gamma$ part, we use

$$
\begin{aligned}
\left(\tilde{\mathfrak{m}}_{\delta} \gamma^{\triangleright}\right)^{2} & \leq\left\|\left(\tau^{0}\right)^{*} \triangleleft \zeta^{\triangleright}\right\|_{E}^{2}+\left|\triangleleft c^{\triangleright}\right|^{2}, \\
\left\|\left(\tau^{0}\right)^{*} \triangleleft \zeta^{\triangleright}\right\|_{E}^{2} & =\left\langle\mathcal{L}^{0} \triangleleft \zeta^{\triangleright} \mid P_{\gamma}^{0} \triangleleft \zeta^{\triangleright}\right\rangle+\left\|P_{d} \gamma^{1}\left(x+\triangleleft c^{\triangleright}\right)\right\|_{E}^{2}
\end{aligned}
$$

with equality at $t=0$. For the distance term, we have from (3.37) and (3.38)

$$
\left|\triangleleft c^{\triangleright}(t)\right| \leq\left|\triangleleft c^{\triangleright}(0)\right|+C \delta \tilde{\mathfrak{m}}_{\delta} v^{\triangleright}(0) .
$$

For the translated part, we have

$$
\begin{aligned}
\left|\left\langle\mathcal{L}^{0} \triangleleft \zeta^{\triangleright} \mid P_{\gamma}^{0} \triangleleft \zeta^{\triangleright}\right\rangle-\left\langle\mathcal{L}^{0} \triangleleft \zeta^{\triangleright} \mid \triangleleft \zeta^{\triangleright}\right\rangle\right| & \lesssim\left\|\triangleleft P_{d}^{\triangleright} \zeta^{1}\right\|_{2}^{2} \lesssim\left|\triangleleft c^{\triangleright}\right|^{2} \delta^{2}, \\
\left\|P_{d} \gamma^{1}\left(x+\triangleleft c^{\triangleright}\right)\right\|_{2}^{2} & \lesssim\left|\triangleleft c^{\triangleright}\right|^{2} \delta^{2},
\end{aligned}
$$

Copyright (c) by SIAM. Unauthorized reproduction of this article is prohibited. 
and

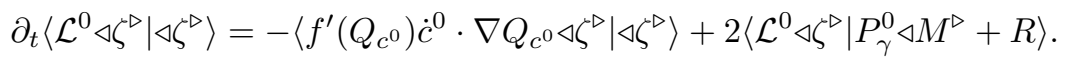

Hence for $|t| \leq 1$, using (3.38) and (3.36) as well,

$$
\begin{aligned}
& \left|\left[\left\langle\mathcal{L}^{0} \triangleleft \zeta^{\triangleright} \mid P_{\gamma}^{0} \triangleleft \zeta^{\triangleright}\right\rangle\right]_{0}^{t}\right| \\
& \quad \lesssim\left|\triangleleft c^{\triangleright}\right|^{2} \delta^{2}+\delta^{2}\left\|\triangleleft \zeta^{\triangleright}\right\|_{L_{t}^{\infty} \mathcal{H}}^{2}+\left\|\triangleleft \zeta^{\triangleright}\right\|_{L_{t}^{\infty} \mathcal{H}}\left\|P_{\gamma}^{0} \triangleleft M^{\triangleright}+R\right\|_{L_{t}^{1} \mathcal{H}} \lesssim \delta^{2}\left(\tilde{\mathfrak{m}}_{\delta} v^{\triangleright}(0)\right)^{2} .
\end{aligned}
$$

Plugging this and (3.40) into (3.39), we obtain the desired upper estimate on the $\gamma$ part. For the lower estimate one reverses time, completing the proof in Case (I).

Case (II): $\min _{\boldsymbol{j}}\left\|\boldsymbol{v}^{\boldsymbol{j}}(\mathbf{0})\right\|_{\mathcal{H}} \geq \boldsymbol{C}_{\mathbf{0}} \boldsymbol{\delta}$. The previous lemma implies that $\left\|v^{j}(t)\right\|_{E} \gtrsim$ $C_{0} \delta \gg \delta$, and so for $|t| \leq 1$ and $j=0,1$,

$$
v^{j}(t)=e^{J \mathcal{L} t} v^{j}(0), \quad\left\|\gamma^{j}(t)\right\|_{E}=\left\|\gamma^{j}(0)\right\|_{E}, \quad\left\|P_{d} \triangleleft v^{\triangleright}(t)\right\|_{E} \lesssim\left\|P_{d} \triangleleft v^{\triangleright}(0)\right\|_{E} .
$$

To estimate the $\gamma$ part, let $\zeta(t):=\gamma^{0}(t)-\gamma^{1}(t, x-q(0))$. Then

$$
\dot{\zeta}-J \mathcal{L} \zeta=\left(0,\left[f^{\prime}(Q)-f^{\prime}\left(Q_{q(0)}\right)\right] \tau_{q(0)} \gamma_{1}^{1}\right),
$$

where the right-hand side is bounded in $\mathcal{H}$ by

$$
|q(0)|\left\|\gamma^{1}(0)\right\|_{\mathcal{H}} \lesssim \delta \tilde{\mathfrak{m}}_{\delta} v^{\triangleright}(0),
$$

where we used that $a \lesssim \delta \phi_{\delta}(a)$. Hence using the energy inequality for $\mathcal{L}$, we get

$$
\begin{aligned}
\left|[\langle\mathcal{L} \zeta \mid \zeta\rangle]_{0}^{t}\right| & \lesssim \delta \tilde{\mathfrak{m}}_{\delta} v^{\triangleright}(0)\|\zeta\|_{L_{t}^{\infty} \mathcal{H}_{x}}, \\
\left\|P_{d} \zeta(t)\right\|_{E} & =\left\|P_{d} \tau_{q(0)} \gamma^{1}\right\|_{E} \lesssim|q(0)|\left\|\gamma^{1}(0)\right\|_{\mathcal{H}} \lesssim \delta \tilde{\mathfrak{m}}_{\delta} v^{\triangleright}(0),
\end{aligned}
$$

and so

$$
\begin{aligned}
\left(\tilde{\mathfrak{m}}_{\delta} \gamma^{\triangleright}(t)\right)^{2} & \leq\|\zeta(t)\|_{E}^{2}+|q(0)|^{2} \phi_{\delta}\left(\left\|\gamma^{1}(0)\right\|_{E}\right)^{2} \\
& \leq\left(\tilde{\mathfrak{m}}_{\delta} \gamma^{\triangleright}(0)\right)^{2}+C \delta^{2}\left(\tilde{\mathfrak{m}}_{\delta} v^{\triangleright}(0)\right)^{2} .
\end{aligned}
$$

This completes the proof in Case (II).

Case (III): $\left\|\boldsymbol{v}^{\mathbf{0}}(\mathbf{0})\right\|_{\mathcal{H}}>\boldsymbol{C}_{\mathbf{1}} \boldsymbol{\delta} \gg \boldsymbol{C}_{\mathbf{0}} \boldsymbol{\delta}>\left\|\boldsymbol{v}^{\mathbf{1}}(\mathbf{0})\right\|_{\mathcal{H}}$. The previous lemma implies that $\left\|v^{0}(t)\right\|_{\mathcal{H}} \gtrsim C_{1} \delta \gg C_{0} \delta \gtrsim\left\|v^{1}(t)\right\|_{\mathcal{H}}$ for $|t| \leq 1$, and so by (2.29),

$$
\tilde{\mathfrak{m}}_{\delta} v^{\triangleright}(t) \simeq\left\|v^{0}(t)\right\|_{\mathcal{H}} \simeq\left\|v^{0}(0)\right\|_{\mathcal{H}} \simeq \tilde{\mathfrak{m}}_{\delta} v^{\triangleright}(0) \gtrsim \delta
$$

For the difference from the linearized solution, (3.15) yields the desired estimate. The estimate on the increment of the $\gamma$ part is similar to Case (I), but now $\zeta^{0}=\gamma^{0}$ evolves linearly, which means that the nonlinear terms in $\triangleleft \zeta^{\triangleright}$ depends only on $\zeta^{1}$. Hence (3.36) is replaced with

$$
\left\|\triangleleft M^{\triangleright}\right\|_{L_{t}^{1} L_{x}^{2}}=\left\|M^{1}\right\|_{L_{t}^{1} L_{x}^{2}} \lesssim\left\|v^{1}\right\|_{\text {Str }}^{2} \lesssim \delta^{2} .
$$

Noting that $\delta \lesssim \tilde{\mathfrak{m}}_{\delta}\left(v^{0}(0), v^{1}(0)\right)$, the rest of the argument goes through as in Case (I).

Case (IV): $\left\|v^{\mathbf{0}}(\mathbf{0})\right\|_{\mathcal{H}}<\boldsymbol{C}_{\mathbf{0}} \delta \ll \boldsymbol{C}_{\mathbf{1}} \boldsymbol{\delta}<\left\|\boldsymbol{v}^{\mathbf{1}}(\mathbf{0})\right\|_{\mathcal{H}}$. Although this is symmetric with the previous case, we have to check the mobile distance part, since there we introduced asymmetry with (3.20). The difference appears in (3.39):

$$
\left(\tilde{\mathfrak{m}}_{\delta} v^{\triangleright}\right)^{2} \leq\left\|\left(\tau^{0}\right)^{*} \triangleleft \zeta^{\triangleright}\right\|_{E}^{2}+\left|\triangleleft c^{\triangleright}\right|^{2} \phi_{\delta}\left(\left\|\gamma^{1}\right\|_{E}\right)^{2} .
$$

However, this is admissible, because

$$
\left[\left|\triangleleft c^{\triangleright}\right| \phi_{\delta}\left(\left\|\gamma^{1}\right\|_{E}\right)\right]_{0}^{t} \lesssim \delta^{2} \phi_{\delta}\left(\left\|v^{1}(0)\right\|_{E}\right) \simeq \delta \tilde{\mathfrak{m}}_{\delta} v^{\triangleright}(0),
$$

and the remaining argument is the same as in the previous case. 
3.3. Evolution of graphs, center-stable case. Now we consider the graphs of $v_{\geq 0} \mapsto v_{-}$satisfying a Lipschitz condition. It is convenient to extend them to the whole $\mathcal{H}$. Our class of graphs for the contraction argument is given by

$$
\mathcal{G}_{\ell, \delta}:=\left\{G: \mathcal{H} \rightarrow P_{-} \mathcal{H} \mid G=G \circ P_{\geq 0}, G(0)=0,\left\|\triangleleft G\left(v^{\triangleright}\right)\right\|_{E} \leq \ell \tilde{\mathfrak{m}}_{\delta} v^{\triangleright}\right\}
$$

for small $\ell>0$, and the graph of $G \in \mathcal{G}_{\ell, \delta}$ is denoted by

$$
\lceil G\rfloor:=\left\{\varphi \in \mathcal{H} \mid P_{-} \varphi=G(\varphi)\right\} .
$$

A center-unstable manifold will be found as the unique invariant graph by the contraction mapping principle in $\mathcal{G}_{\ell, \delta}$.

For $p>d /(d-2)$, the Sobolev inequality does not imply that $\dot{v}$ is bounded in $L_{x}^{2}$, and consequently we cannot prove strict invariance of $\mathcal{G}_{\ell, \delta}$ for $t>0$, but the "almost invariance" given below is sufficient for the contraction argument.

Lemma 3.3. There exists $C_{L} \geq 1$ such that if $\ell, \delta>0$ satisfy

$$
\bar{k} \ell^{2}+\delta \ll \underline{k}, \quad \delta \ll \ell \underline{k},
$$

then for any two solutions $v^{j}(t)=U(t) v^{j}(0)(j=0,1)$ satisfying

$$
\left\|\triangleleft v_{-}^{\triangleright}(0)\right\|_{E} \leq \ell \tilde{\mathfrak{m}}_{\delta} v^{\triangleright}(0),
$$

one has

$$
\left\|\triangleleft v_{-}^{\triangleright}(t)\right\|_{E} \leq \begin{cases}C_{L} \ell \tilde{\mathfrak{m}}_{\delta} v^{\triangleright}(t) & (|t| \leq 1), \\ \ell \tilde{\mathfrak{m}}_{\delta} v^{\triangleright}(t) & (1 / 2 \leq t \leq 1) .\end{cases}
$$

Proof. The linearized solutions of the discrete modes are estimated by

$$
\begin{aligned}
\min \left(e^{ \pm \underline{k} t}, e^{ \pm \bar{k} t}\right)\left\|P_{ \pm} \varphi\right\|_{E} & \leq\left\|P_{ \pm} e^{J \mathcal{L} t} \varphi\right\|_{E} \leq \max \left(e^{ \pm \underline{k} t}, e^{ \pm \bar{k} t}\right)\left\|P_{ \pm} \varphi\right\|_{E} \\
e^{-\kappa|t|}\left\|P_{0} \varphi\right\|_{E} & \leq\left\|P_{0} e^{J \mathcal{L} t} \varphi\right\|_{E} \leq e^{\kappa|t|}\left\|P_{0} \varphi\right\|_{E}
\end{aligned}
$$

The previous lemma implies that

$$
\begin{aligned}
\left\|\triangleleft v_{-}^{\triangleright}(t)\right\|_{E} & \leq\left\|\triangleleft e^{J \mathcal{L} t} v_{-}^{\triangleright}(0)\right\|_{E}+C \delta \tilde{\mathfrak{m}}_{\delta} v^{\triangleright}(0) \\
& \leq\left[\max \left(e^{-\underline{k} t}, e^{-\bar{k} t}\right) \ell+C \delta\right] \tilde{\mathfrak{m}}_{\delta} v^{\triangleright}(0),
\end{aligned}
$$

and also

$$
\tilde{\mathfrak{m}}_{\delta} v^{\triangleright}(t)^{2} \geq\left\|e^{J \mathcal{L} t} \triangleleft v_{d}^{\triangleright}(0)\right\|_{E}^{2}+\tilde{\mathfrak{m}}_{\delta} \gamma^{\triangleright}(0)^{2}-C \delta^{2} \tilde{\mathfrak{m}}_{\delta} v^{\triangleright}(0)^{2} .
$$

Plugging (3.58) into the last estimate, we obtain

$$
\tilde{\mathfrak{m}}_{\delta} v^{\triangleright}(t)^{2} \geq \begin{cases}{\left[e^{-2 \kappa t}\left(1-\ell^{2}\right)+e^{-2 \bar{k} t} \ell^{2}-C \delta^{2}\right] \tilde{\mathfrak{m}}_{\delta} v^{\triangleright}(0)^{2}} & (0 \leq t \leq 1), \\ {\left[e^{-2 \bar{k}|t|}-C \delta^{2}\right] \tilde{\mathfrak{m}}_{\delta} v^{\triangleright}(0)^{2}} & (|t| \leq 1) .\end{cases}
$$

Combining it with (3.59) yields for $|t| \leq 1$,

$$
\left\|\triangleleft v_{-}^{\triangleright}(t)\right\|_{E} \leq\left(e^{\bar{k}} \ell+C \delta\right)\left(e^{\bar{k}}+C \delta\right) \tilde{\mathfrak{m}}_{\delta} v^{\triangleright}(t) \leq 2 \ell e^{2 \bar{k}} \tilde{\mathfrak{m}}_{\delta} v^{\triangleright}(t),
$$

Copyright (c) by SIAM. Unauthorized reproduction of this article is prohibited. 
provided that $\delta \ll \ell \ll 1$. For $1 / 2 \leq t \leq 1$, we obtain

$$
\begin{aligned}
\left\|\triangleleft v_{-}^{\triangleright}(t)\right\|_{E} & \leq\left(e^{-\underline{k} / 2} \ell+C \delta\right)\left(e^{-2 \kappa}\left(1-\ell^{2}\right)+e^{-2 \bar{k}} \ell^{2}-C \delta^{2}\right)^{-1 / 2} \tilde{\mathfrak{m}}_{\delta} v^{\triangleright} \\
& \leq(1-\underline{k} / 3+C \delta / \ell) \ell\left[1+C\left(\delta+\kappa+\ell^{2} \underline{k}\right)\right] \tilde{\mathfrak{m}}_{\delta} v^{\triangleright} \leq \ell \tilde{\mathfrak{m}}_{\delta} v^{\triangleright}
\end{aligned}
$$

under the condition (3.55) and $\kappa \ll \underline{k} \leq 1$.

As an immediate consequence of the above lemma together with a mapping degree argument, we obtain the following result.

LEMMA 3.4. Under the condition (3.55), $U(t)$ for $|t| \leq 1$ defines a map $\mathcal{U}(t)$ : $\mathcal{G}_{\ell, \delta} \rightarrow \mathcal{G}_{C_{L} \ell, \delta}$ uniquely by the relation $U(t)\lceil G\rfloor=\lceil\mathcal{U}(t) G\rfloor$. Moreover, if $1 / 2 \leq t \leq 1$, then $\mathcal{U}(t)$ maps $\mathcal{G}_{\ell, \delta}$ into itself.

Proof. The previous lemma yields for any $\varphi^{0}, \varphi^{1} \in U(t)\lceil G\rfloor$,

$$
\left\|\triangleleft \varphi_{-}^{\triangleright}\right\|_{E} \lesssim \ell \tilde{\mathfrak{m}}_{\delta} \varphi^{\triangleright} .
$$

Since $\ell \ll 1$, it implies $\left\|\triangleleft \varphi_{-}^{\triangleright}\right\|_{E} \ll \tilde{\mathfrak{m}}_{\delta} \varphi_{>0}^{\triangleright}$. Then the conditions $U(t)\lceil G\rfloor \subset\lceil\mathcal{U}(t) G\rfloor$ and $\mathcal{U}(t) G \circ P_{\geq 0}=\mathcal{U}(t) G$ define $\mathcal{U}(t) G$ uniquely and consistently on the set

$$
P_{\geq 0} U(t)\lceil G\rfloor+P_{-} \mathcal{H} \text {. }
$$

The proof is complete once the above is shown to be $\mathcal{H}$, for which we use the degree argument. Suppose for contradiction that there exists $\psi \in P_{\geq 0} \mathcal{H} \backslash P_{\geq 0} U(t)\lceil G\rfloor$. In other words, for any $a \in P_{-} \mathcal{H}$,

$$
U(-t)(a+\psi) \notin\lceil G\rfloor .
$$

Let $m(a):=P_{-} U(-t)(a+\psi)-G(U(-t)(a+\psi))$; then $m$ is a continuous map from $P_{-} \mathcal{H}$ to itself, such that $0 \notin m\left(P_{-} \mathcal{H}\right)$. On the other hand, if $|a| \gg \delta$, then

$$
m(a)=e^{-J \mathcal{L} t} a-G\left(e^{-J \mathcal{L} t} \psi\right) .
$$

Define $\Phi: \mathbb{R}^{K} \rightarrow P_{-} \mathcal{H}, \Psi: \mathbb{R}^{K} \backslash\{0\} \rightarrow S^{K-1}$ and $\widetilde{m}:(0, \infty) \times S^{K-1} \rightarrow S^{K-1}$ by

$$
\Phi(X)=\sum_{k \in K} X_{k} g_{k-}, \quad \Psi(X)=\frac{X}{|X|}, \quad \widetilde{m}(R, \theta)=\Psi \circ \Phi^{-1} \circ m \circ \Phi(R \theta) .
$$

Then $\widetilde{m}$ is continuous, but the degree of $\widetilde{m}(R, \cdot)$ is 0 for small $R>0$ and 1 for large $R$, which is a contradiction. Hence $\mathcal{U}(t) G$ is well-defined as a map on $\mathcal{H}$ which is right-invariant for $P_{\geq 0}$. The Lipschitz bounds are immediate from the previous lemma.

3.4. Contraction of graphs, center-stable case. We introduce the following norm in $\mathcal{G}:=\bigcup_{\ell>0} \mathcal{G}_{\ell, \delta}:$

$$
\|G\|_{\mathcal{G}}:=\sup _{\psi \in \mathcal{H}} \frac{\|G(\psi)\|_{E}}{\|\psi\|_{E}} .
$$

It is easy to see that the set $\mathcal{G}$ is independent of $\delta>0$. For any $G \in \mathcal{G}_{\ell, \delta}$ and any $\psi \in \mathcal{H}$, we have $\|G(\psi)\|_{E} \leq \ell \tilde{\mathfrak{m}}_{\delta}(\psi, 0) \leq \ell\|\psi\|_{E}$, and so

$$
\|G\|_{\mathcal{G}} \leq \ell .
$$

$\mathcal{G}$ is a Banach space with this norm, where each $\mathcal{G}_{\ell, \delta}$ is a bounded closed set. The contraction argument is completed by the next lemma. 
Lemma 3.5. In addition to (3.55), let

$$
\delta \ll \underline{k}^{2} .
$$

Then the map $\mathcal{U}(t)$ is a contraction on $\mathcal{G}_{\ell, \delta}$ for all $t \geq 1 / 2$.

Proof. Let $T \in[1 / 2,1]$. For any $G^{j} \in \mathcal{G}_{\ell, \delta}$ for $j=0,1$ and any $\psi \in \mathcal{H}$, let

$$
v^{j}(t):=U(t-T)\left[P_{\geq 0} \psi+\left(\mathcal{U}(T) G^{j}\right) \psi\right] .
$$

Since $P_{\geq 0} v^{0}(T)=P_{\geq 0} v^{1}(T)$, we have

$$
\tilde{\mathfrak{m}}_{\delta} v^{\triangleright}(T)=\left\|\triangleleft v_{-}^{\triangleright}(T)\right\|_{E} .
$$

Applying Lemma 3.2 from $t=T$, we get for $0 \leq t \leq T$,

$$
\left\|\triangleleft v_{d}^{\triangleright}(t)-e^{J \mathcal{L}(t-T)} \triangleleft v_{-}^{\triangleright}(T)\right\|_{E}+\tilde{\mathfrak{m}}_{\delta} \gamma^{\triangleright}(t) \lesssim \delta\left\|\triangleleft v_{-}^{\triangleright}(T)\right\|_{E} .
$$

Hence using the same estimate on the linearized solution as in (3.58)

$$
\begin{aligned}
\left\|\triangleleft v_{-}^{\triangleright}(0)\right\|_{E} & \geq\left\|e^{-J \mathcal{L} T_{\triangleleft}} v_{-}^{\triangleright}(T)\right\|_{E}-C \delta\left\|\triangleleft v_{-}^{\triangleright}(T)\right\|_{E} \geq\left(e^{\underline{\underline{k}} T}-C \delta\right)\left\|\triangleleft v_{-}^{\triangleright}(T)\right\|_{E}, \\
\tilde{\mathfrak{m}}_{\delta} v_{\geq 0}^{\triangleright}(0) & \lesssim \delta\left\|\triangleleft v_{-}^{\triangleright}(T)\right\|_{E} .
\end{aligned}
$$

On the other hand, since $v_{-}^{j}(0)=G^{j}\left(v^{j}(0)\right)$ and $G^{j} \in \mathcal{G}_{\ell, \delta}$,

$$
\begin{aligned}
\left\|\triangleleft v_{-}^{\triangleright}(0)\right\|_{E} & \leq\left\|\triangleleft G^{\triangleright}\left(v^{0}(0)\right)\right\|_{E}+\left\|\triangleleft G^{1}\left(v^{\triangleright}(0)\right)\right\|_{E} \\
& \leq\left\|\triangleleft G^{\triangleright}\right\|_{\mathcal{G}}\left\|v_{\geq 0}^{0}(0)\right\|_{E}+\ell \tilde{\mathfrak{m}}_{\delta} v_{\geq 0}^{\triangleright}(0) .
\end{aligned}
$$

Equation (3.4) as well as (3.58) yields

$$
\left\|v_{\geq 0}^{0}(0)\right\|_{E}^{2} \leq\left(e^{2 \kappa T}+C \delta\right)\|\psi\|_{E}^{2} .
$$

Inserting this and the second inequality of (3.75) into (3.76), we obtain

$$
\left\|\triangleleft v_{-}^{\triangleright}(0)\right\|_{E} \leq\left(e^{\kappa T}+C \sqrt{\delta}\right)\left\|\triangleleft G^{\triangleright}\right\|_{\mathcal{G}}\|\psi\|_{E}+\ell \delta\left\|\triangleleft v_{-}^{\triangleright}(T)\right\|_{E} .
$$

Combining this and (3.75), we conclude that

$$
\begin{aligned}
\left\|\triangleleft v_{-}^{\triangleright}(T)\right\|_{E} & \leq(1-C \delta \ell)^{-1}\left(e^{\underline{k} T}-C \delta\right)^{-1}\left(e^{\kappa T}+C \sqrt{\delta}\right)\left\|\triangleleft G^{\triangleright}\right\|_{\mathcal{G}}\|\psi\|_{E} \\
& \leq e^{-(\underline{k}-\kappa) T}(1+C \sqrt{\delta})\left\|\triangleleft G^{\triangleright}\right\|_{\mathcal{G}}\|\psi\|_{E} .
\end{aligned}
$$

Equation (3.71) and $\kappa \ll \underline{k}$ imply that there exists $\Lambda<1$, determined by $\underline{k}, \kappa, \delta, \ell$ such that

$$
\frac{\left\|\triangleleft v_{-}^{\triangleright}(T)\right\|_{E}}{\|\psi\|_{E}} \leq \Lambda\left\|\triangleleft G^{\triangleright}\right\|_{\mathcal{G}} .
$$

Taking the supremum over all $\psi \in \mathcal{H}$ yields

$$
\left\|\triangleleft \mathcal{U}(T) G^{\triangleright}\right\|_{\mathcal{G}} \leq \Lambda\left\|\triangleleft G^{\triangleright}\right\|_{\mathcal{G}},
$$

as desired. The case $T>1$ is now obvious by iteration.

Thus we obtain the next theorem.

Copyright (c) by SIAM. Unauthorized reproduction of this article is prohibited. 
TheOREm 3.6. Suppose that $\ell, \delta>0$ satisfy (3.55) and (3.71). Then there exists a unique $G_{*} \in \mathcal{G}_{\ell, \delta}$ such that $\mathcal{U}(t) G_{*}=G_{*}$ for all $t \geq 0$. The uniqueness holds for any fixed $t>0$.

Proof. For any $T \geq 1 / 2$, the above lemma implies that there is a unique fixed point of $\mathcal{U}(T)$ in $\mathcal{G}_{\ell, \delta}$. Since the equation is invariant for time translation, it implies that $\mathcal{U}(t) G \in \mathcal{G}_{C_{L} \ell, \delta}$ is also a fixed point for all $0 \leq t \leq 1$. Then the uniqueness of the fixed point implies that $\mathcal{U}(t) G=G$ for all $0 \leq t \leq 1$ and so for all $t \geq 0$. If $\mathcal{U}(t) H=H$ for some $t>0$ and some $H \in \mathcal{G}_{\ell, \delta}$, then by iteration $\mathcal{U}(T) H=H$ for some $T \geq 1 / 2$, and so $H=G$.

Since $U(t)$ is invertible, $U(t)\left\lceil G_{*}\right\rfloor=\left\lceil G_{*}\right\rfloor$ for all $t \in \mathbb{R}$.

The conditions (3.55) and (3.71) are satisfied for $\ell=O(\delta)$ as $\delta \rightarrow+0$, which implies that

$$
\left\lceil G_{*}\right\rfloor \ni \varphi,\|\varphi\|_{\mathcal{H}} \leq \delta \Longrightarrow\left|\omega\left(\varphi, g_{k+}\right)\right| \lesssim \delta^{2} ;
$$

in other words, $\left\lceil G_{*}\right\rfloor$ is normal at 0 to $(-k, 1) \rho_{k}$ for each $k \in K$.

Notice that the above construction did not really use the special property of the generalized null space of the linearized operator. However, the constructed manifold makes sense for the original equation only on the subset $\mathcal{H}_{\perp}$, for which we need the property that the generalized null space is exactly generated by the symmetries of the equation.

3.5. Evolution of graphs, unstable case. We now carry out an analogous procedure for the finite-dimensional unstable manifold. Thus, we now consider the graphs of $v_{+} \mapsto v_{\leq 0}$ satisfying a Lipschitz condition

$$
\mathcal{G}_{\ell, \delta}^{+}:=\left\{G: \mathcal{H} \rightarrow P_{\leq 0} \mathcal{H} \mid G=G \circ P_{+}, G(0)=0, \tilde{\mathfrak{m}}_{\delta} G\left(v^{\triangleright}\right) \leq \ell\left\|\triangleleft v_{+}^{\triangleright}\right\|_{E}\right\}
$$

for small $\ell>0$, and the graph of $G \in \mathcal{G}_{\ell, \delta}^{+}$is denoted by

$$
\lceil G\rfloor:=\left\{\varphi \in \mathcal{H} \mid P_{\leq 0} \varphi=G(\varphi)\right\} .
$$

The unstable manifold will be found as the unique invariant graph by the contraction mapping principle in $\mathcal{G}_{\ell, \delta}^{+}$. We formulate the analogue of Lemma 3.3 in this case.

LEMma 3.7. There exists $C_{L} \geq 1$ such that if $\ell, \delta>0$ satisfy (3.55), then for any two solutions $v^{j}(t)=U(t) v^{j}(0)(j=0,1)$ satisfying

$$
\tilde{\mathfrak{m}}_{\delta} v_{\leq 0}^{\triangleright}(0) \leq \ell\left\|\triangleleft v_{+}^{\triangleright}(0)\right\|_{E},
$$

one has

$$
\tilde{\mathfrak{m}}_{\delta} v_{\leq 0}^{\triangleright}(t) \leq \begin{cases}C_{L} \ell\left\|\triangleleft v_{+}^{\triangleright}(t)\right\|_{E} & (|t| \leq 1), \\ \ell\left\|\triangleleft v_{+}^{\triangleright}(t)\right\|_{E} & (1 / 2 \leq t \leq 1) .\end{cases}
$$

Proof. We again have (3.58) for the linearized solutions of the discrete modes. In particular,

$$
\left\|\triangleleft v_{+}^{\triangleright}(t)\right\|_{E} \geq \min \left(e^{\underline{k} t}, e^{\bar{k} t}\right)\left\|\triangleleft v_{+}^{\triangleright}(0)\right\|_{E}-C \delta \tilde{\mathfrak{m}}_{\delta} v^{\triangleright}(0) .
$$

By Lemma 3.2, for $t \geq 0$,

$$
\begin{aligned}
\tilde{\mathfrak{m}}_{\delta} v_{\leq 0}^{\triangleright}(t)^{2} & \leq\left\|\triangleleft e^{J \mathcal{L} t} v_{0-}^{\triangleright}(0)\right\|_{E}^{2}+\tilde{\mathfrak{m}}_{\delta} \gamma^{\triangleright}(0)^{2}+C \delta^{2} \tilde{\mathfrak{m}}_{\delta} v^{\triangleright}(0)^{2} \\
& \leq\left(e^{2 \kappa t} \ell^{2}+C \delta^{2}\left(1+\ell^{2}\right)+\ell^{2}\right)\left\|\triangleleft v_{+}^{\triangleright}(0)\right\|_{E}^{2}
\end{aligned}
$$

and one now concludes by combining these estimates; cf. Lemma 3.3. 
One now has the following analogue of Lemma 3.4.

Lemma 3.8. Under condition (3.55), $U(t)$ for $|t| \leq 1$ defines a map $\mathcal{U}(t): \mathcal{G}_{\ell, \delta}^{+} \rightarrow$ $\mathcal{G}_{C_{L} \ell, \delta}^{+}$uniquely by the relation $U(t)\lceil G\rfloor=\lceil\mathcal{U}(t) G\rfloor$. Moreover, if $t \geq \frac{1}{2}$, then $\mathcal{U}(t)$ maps $\mathcal{G}_{\ell, \delta}^{+}$into itself.

Proof. The mapping properties for $|t| \leq 1$ and $\frac{1}{2} \leq t \leq 1$ are an immediate consequence of the previous lemma. The extension to $t \geq \frac{1}{2}$ then follows by iteration. As in the case of the center-stable version, the main issue is to show that $P_{+} U(t)\lceil G\rfloor=$ $P_{+} \mathcal{H}=\mathcal{H}_{+}$for all $|t| \leq 1$. Thus take $\psi_{0} \in \mathcal{H}_{+}$and denote the $R$-ball in $\mathcal{H}_{+}$by $B_{R}^{+}$. Lemma 3.2 implies that if $0<\ell \ll 1$, then

$$
\left\|P_{+} U(t)(\psi+G(\psi))\right\|_{\mathcal{H}} \gtrsim R \quad \forall|t| \leq 1, \forall \psi \in \partial B_{R}^{+}
$$

for any $G \in \mathcal{G}_{\ell, \delta}^{+}$and with absolute implicit constants. This shows that with $\Phi(\psi)=$ $\psi+G(\psi)$,

$$
\operatorname{deg}\left(P_{+} U(t) \Phi, B_{R}^{+}, \psi_{0}\right)=1 \quad \forall|t| \leq 1
$$

provided $R$ is sufficiently large, and we are done.

3.6. Contraction of graphs, unstable case. Let $\mathcal{G}^{+}:=\bigcup_{\ell>0} \mathcal{G}_{\ell, \delta}^{+}$. As before, the set $\mathcal{G}^{+}$is independent of $\delta>0$. We introduce the following quasi-distance $d_{+}$in $\mathcal{G}^{+}$: for any $G^{1}, G^{2} \in \mathcal{G}^{+}$let

$$
d_{+}\left(G^{1}, G^{2}\right):=\sup _{\psi \in \mathcal{H}} \frac{\tilde{\mathfrak{m}}_{\delta} G^{\triangleright}(\psi)}{\|\psi\|_{E}} .
$$

It is clear that this expression is finite and that it satisfies a triangle inequality with the same multiplicative loss as in Proposition 2.2:

$$
d_{+}\left(G^{1}, G^{3}\right) \leq C_{d}\left(d_{+}\left(G^{1}, G^{2}\right)+d_{+}\left(G^{2}, G^{3}\right)\right)
$$

Moreover, $\mathcal{G}^{+}$is a complete quasi-distance space, in which $\mathcal{G}_{\ell, \delta}^{+}$is closed. Recall that the Banach fixed point theorem is valid in complete quasi-distance spaces.

Lemma 3.9. Let $X$ be a complete quasi-distance space, and let $A: X \rightarrow X$ be a contraction. Then there is a unique fixed point $x_{*} \in X$ of $A$, which is obtained by $x_{*}=\lim _{n \rightarrow \infty} A^{n}(x)$ for any $x \in X$.

Proof. Let $C \geq 1$ be the constant in the quasi-triangle inequality in $X$, let $\Lambda \in(0,1)$ be the Lipschitz constant of $A$, and fix $m \in \mathbb{N}$ so that $\Lambda^{m} C<1$. Take any $x_{0} \in X$ and let $x_{n}=A^{n m}\left(x_{0}\right)$ for each $n \in \mathbb{N}$. Then

$$
d\left(x_{n+1}, x_{n}\right)=d\left(A^{m}\left(x_{n}\right), A^{m}\left(x_{n-1}\right)\right) \leq \Lambda^{m} d\left(x_{n}, x_{n-1}\right) \leq \cdots \leq \Lambda^{m n} d\left(x_{1}, x_{0}\right) .
$$

Hence for any $k>j \geq 1$, by repeated use of the quasi-triangle inequality,

$$
\begin{aligned}
d\left(x_{k}, x_{j}\right) & \leq C d\left(x_{k}, x_{j+1}\right)+C d\left(x_{j+1}, x_{j}\right) \leq \cdots \leq \sum_{l=j+1}^{k} C^{l-j} d\left(x_{l}, x_{l-1}\right) \\
& \leq \sum_{l=j+1}^{k}\left(C \Lambda^{m}\right)^{l-j} \Lambda^{m j} d\left(x_{1}, x_{0}\right) \leq \frac{\Lambda^{m j}}{1-C \Lambda^{m}} d\left(x_{1}, x_{0}\right) .
\end{aligned}
$$

Hence $x_{n} \rightarrow \exists x_{\infty} \in X$, and by the continuity of $A, A^{m}\left(x_{\infty}\right)=x_{\infty}$. Then

$$
d\left(A\left(x_{\infty}\right), x_{\infty}\right)=d\left(A^{m+1}\left(x_{\infty}\right), A^{m}\left(x_{\infty}\right)\right) \leq \Lambda^{m} d\left(A\left(x_{\infty}\right), x_{\infty}\right)
$$

which implies that $A\left(x_{\infty}\right)=x_{\infty}$. The uniqueness follows in the well-known way. 
The following is an analogue of Lemma 3.5, but here the evolution time has to be long enough to absorb the quasi-triangle factor $C_{d}$ in using the "chain-rule" in $\mathcal{G}^{+}$.

LEMma 3.10. There are $\delta_{0}>0$ and $T>0$ such that if $\delta \leq \delta_{0}$ and (3.55) is satisfied, then the map $\mathcal{U}(t)$ is a contraction on $\mathcal{G}_{\ell, \delta}^{+}$for all $t \geq T$.

Proof. Let $G^{1}, G^{2} \in \mathcal{G}_{\ell, \delta}^{+}, T>0, \psi \in \mathcal{H}_{+}$, and

$$
v^{j}(t):=U(t-T)\left[\psi+\left(\mathcal{U}(T) G^{j}\right) \psi\right] \quad(j=0,1) .
$$

Iterating Lemmas 3.1 and 3.2 from $t=T$ down to $t=0$, we obtain

$$
\left\|v^{\triangleright}(t)\right\|_{E} \leq C e^{C T}\|\psi\|_{E}, \quad \tilde{\mathfrak{m}}_{\delta} v^{\triangleright}(t) \leq C e^{C T} \tilde{\mathfrak{m}}_{\delta} v^{\triangleright}(T)=C e^{C T} \tilde{\mathfrak{m}}_{\delta} v_{\leq 0}^{\triangleright}(T)
$$

for $0 \leq t \leq T$ with some constant $C \geq 1$ (determined by $d, Q, f$, and $\kappa$ ). Hence if

$$
\delta \ll e^{-2 C T} / C^{2},
$$

then by iteration of those lemmas again, we deduce that

$$
\begin{aligned}
& \left\|v_{+}^{0}(0)\right\|_{E} \lesssim e^{-\underline{k} T / 2}\|\psi\|_{E}, \\
& \tilde{\mathfrak{m}}_{\delta} v_{\leq 0}^{\triangleright}(0) \geq e^{-2 \kappa T} \tilde{\mathfrak{m}}_{\delta} v_{\leq 0}^{\triangleright}(T), \quad\left\|\triangleleft v_{+}^{\triangleright}(0)\right\|_{E} \lesssim \sqrt{\delta} \tilde{\mathfrak{m}}_{\delta} v_{\leq 0}^{\triangleright}(T) .
\end{aligned}
$$

Since $v_{\leq 0}^{j}(0)=G^{j}\left(v^{j}(0)\right)$ and $G^{j} \in \mathcal{G}_{\ell, \delta}^{+}$,

$$
\begin{aligned}
\tilde{\mathfrak{m}}_{\delta} v_{\leq 0}^{\triangleright}(0) & \leq C_{d}\left[\tilde{\mathfrak{m}}_{\delta} G^{\triangleright}\left(v^{0}(0)\right)+\tilde{\mathfrak{m}}_{\delta} G^{1}\left(v^{\triangleright}(0)\right)\right] \\
& \leq C_{d}\left[d_{+}\left(G^{\triangleright}\right)\left\|v_{+}^{0}(0)\right\|_{E}+\ell\left\|\triangleleft v_{+}^{\triangleright}(0)\right\|_{E}\right] .
\end{aligned}
$$

Plugging (3.97) into the above, we obtain

$$
\tilde{\mathfrak{m}}_{\delta} v_{\leq 0}^{\triangleright}(T) \leq e^{2 \kappa T} C_{d}\left[d_{+}\left(G^{\triangleright}\right) C e^{-\underline{k} T / 2}\|\psi\|_{E}+\ell \sqrt{\delta} \tilde{\mathfrak{m}}_{\delta} v_{\leq 0}^{\triangleright}(T)\right] .
$$

Choosing $T$ so large while keeping (3.96), we can ensure that

$$
\tilde{\mathfrak{m}}_{\delta}\left(\mathcal{U}(T) G^{\triangleright}\right) \psi=\tilde{\mathfrak{m}}_{\delta} v_{\leq 0}^{\triangleright}(T) \leq \Lambda\|\psi\|_{E}
$$

for some constant $\Lambda \in(0,1)$ determined by $d, f, Q, \kappa, T$, and $\delta$. Obviously, this remains true even if we replace $T$ with any $T^{\prime} \in[T, 2 T]$, taking $\delta$ even smaller if necessary. Hence iterating $\mathcal{U}(t)$ allows one to draw the same conclusion for all $t \geq T$.

By the same arguments as in Theorem 3.6 one now concludes the following.

THEOREM 3.11. Suppose that $\ell, \delta>0$ satisfy the assumptions of the previous lemma. Then there exists a unique $G_{*}^{+} \in \mathcal{G}_{\ell, \delta}^{+}$such that $\mathcal{U}(t) G_{*}^{+}=G_{*}^{+}$for all $t \geq 0$. The uniqueness holds for any fixed $t>0$. Moreover, if $v(0) \in\left\lceil G_{*}^{+}\right\rfloor$, then $\|U(t) v(0)\|_{\mathcal{H}} \rightarrow 0$ exponentially as $t \rightarrow-\infty$; in fact, for any $\varepsilon>0$,

$$
e^{-(\underline{k}-\varepsilon) t}\|U(t) v(0)\|_{\mathcal{H}} \rightarrow 0, \quad t \rightarrow-\infty .
$$

Proof. The estimate (3.101) follows from the previous proof. In fact, (3.97) implies

$$
\|v(t)\|_{E} \lesssim e^{\frac{k}{2} t}\|v(0)\|_{E} \quad(t \rightarrow \infty)
$$

where $v(t):=U(t) v(0)$, but we could take the exponent arbitrarily close to $\underline{k}$ by choosing $\delta$ even smaller. Since $v(t)$ comes into any $\delta$ ball as $t \rightarrow-\infty$, we may apply such decay estimates for $t$ sufficiently close to $-\infty$, thereby deducing (3.101) for all $\varepsilon>0$. 
4. The unstable manifold. We now describe the unstable manifold in the original $u$-formulation of the equation; see (1.1). Let $G_{*}^{+}$be as in Theorem 3.11. For any $v(0) \in\left\lceil\mathcal{G}_{\ell, \delta}^{+}\right\rfloor$with $\|v(0)\|_{\mathcal{H}}<\delta$, define $v(t)=U(t) v(0)$,

$$
u(t)=(\vec{Q}+v)(t, \cdot-c(t)), \quad c(0)=c_{0}, \quad \dot{c}(t)=A(v(t)),
$$

where $c_{0} \in \mathbb{R}^{d}$ is a fixed vector. By construction, $u$ solves (1.1), and by (3.101) one has $\dot{c}(t) \rightarrow 0$ and $c(t) \rightarrow c(-\infty)$ exponentially fast as $t \rightarrow-\infty$. In particular, $u$ has vanishing momentum: $P(u)=0$. Then by design (cf. $(2.24)-(2.26)), \omega(v, J \nabla \vec{Q})$ is constant, and since it converges to zero as $t \rightarrow-\infty$, it must vanish. To summarize, we have obtained the following characterization of the unstable manifold.

COROLlary 4.1. The unstable manifold $\mathcal{M}_{u}$ is the set of all $\vec{u}(0)$ with $u$ defined in terms of $G_{*}^{+}$by means of (4.1). $\mathcal{M}_{u}$ is invariant in backward time, and all solutions starting in $\mathcal{M}_{u}$ converge to a trajectory of the form $\vec{Q}(\cdot-c(t))$ exponentially fast as $t \rightarrow-\infty$ with $\dot{c}(t) \rightarrow 0$ as $t \rightarrow-\infty$ exponentially fast. $\mathcal{M}_{u}$ is a Lipschitz manifold of dimension $K+d$.

The dimension count is a result of the fact that $\left[G_{*}^{+}\right\rfloor$is of dimension $K$, and the translations (see $c_{0}$ in (4.1)) add another $d$ dimensions.

\section{Trapping property of the center-stable manifold.}

5.1. Restriction by the orthogonality. For any Banach space $X$, denote the ball around 0 of radius $R>0$ by

$$
B_{R}(X):=\{\varphi \in X \mid\|\varphi\|<R\} .
$$

Lemma 5.1. If $\ell \leq 1$, and $\delta>0$ is small enough (depending only on $d, f, Q$ ), then for any $G \in \mathcal{G}_{\ell, \delta}$, there is a unique map $\widetilde{G}: P_{\gamma+} B_{\delta}(\mathcal{H}) \rightarrow P_{0-} \mathcal{H}$ such that

$$
\begin{aligned}
\lceil\widetilde{G}\rfloor: & =\left\{\psi+\widetilde{G}(\psi) \mid \psi \in P_{\gamma+} B_{\delta}(\mathcal{H})\right\} \\
& =\left\{\varphi \in\lceil G\rfloor \cap \mathcal{H}_{\perp}\left|P_{\gamma+} \varphi \in P_{\gamma+} B_{\delta}(\mathcal{H}),\right| P_{\nu} \varphi \mid<\delta\right\} .
\end{aligned}
$$

Moreover, $\widetilde{G}$ is Lipschitz continuous in the mobile distance $\tilde{\mathfrak{m}}_{\delta}$.

Proof. For any $\psi \in \mathcal{H}$ and $\nu \in \mathbb{R}^{d}$, put

$$
\widetilde{\psi}(\nu):=\psi+\nu \cdot J \nabla \vec{Q}, \quad \widetilde{\varphi}(\nu):=\widetilde{\psi}(\nu)+G(\widetilde{\psi}(\nu)) .
$$

It suffices to show that for any $\psi \in P_{\gamma+} B_{\delta}(\mathcal{H})$, there is a unique fixed point $\nu \in B_{\delta}\left(\mathbb{R}^{d}\right)$ for the map

$$
\nu \mapsto \mathcal{N}(\nu):=H(Q)^{-1} \omega(\widetilde{\varphi}(\nu), \nabla \widetilde{\varphi}(\nu)) / 2 .
$$

For any $\nu^{j} \in B_{\delta}\left(\mathbb{R}^{d}\right)(j=0,1)$, we have

$$
\triangleleft \widetilde{\varphi}\left(\nu^{\triangleright}\right)=\triangleleft \nu^{\triangleright} \cdot J \nabla \vec{Q}+\triangleleft G\left(\psi+\nu^{\triangleright} \cdot J \nabla \vec{Q}\right) .
$$

Hence using $\|\psi\|_{\mathcal{H}}<\delta$ and $G \in \mathcal{G}_{\ell, \delta}$ as well, we deduce that

$$
|\mathcal{N}(\nu)| \lesssim\|\widetilde{\varphi}(\nu)\|_{\mathcal{H}}^{2} \lesssim \delta^{2}, \quad\left|\triangleleft \omega\left(\widetilde{\varphi}\left(\nu^{\triangleright}\right), \nabla \widetilde{\varphi}\left(\nu^{\triangleright}\right)\right)\right| \lesssim \delta\left|\triangleleft \nu^{\triangleright}\right| .
$$

Therefore $\mathcal{N}: B_{\delta}\left(\mathbb{R}^{d}\right) \rightarrow B_{\delta}\left(\mathbb{R}^{d}\right)$ is a contraction for small $\delta, \ell>0$, and so has a unique fixed point $\nu=\mathcal{N}(\nu) \in B_{\delta}\left(\mathbb{R}^{d}\right)$. Since $\mathcal{N}$ is Lipschitz for $\psi$ in the mobile distance, so is the fixed point $\nu(\psi)$, as well as $\widetilde{G}$.

Hence $\lceil G\rfloor \cap \mathcal{H}_{\perp}$ is a Lipschitz manifold in the mobile distance around 0 with codimension $K+2 d=\operatorname{dim} P_{0-} \mathcal{H}$. 
5.2. Solutions on the center-stable manifold with the orthogonality. Let $G=G_{*} \in \mathcal{G}_{\ell, \delta}$ be the map for the center-unstable manifold of the localized equation given by Theorem 3.6, and let $\widetilde{G}=\widetilde{G}_{*}$ be the map for its orthogonal restriction given by the above lemma. The invariance of $\lceil G\rfloor$ means that for any $v(0) \in\lceil G\rfloor$, $v(t):=U(t) v(0)$ stays on $\lceil G\rfloor$ for all $t \in \mathbb{R}$. Let $c(t)$ be the solution of

$$
c(0)=0, \quad \dot{c}(t)=A(v(t)),
$$

and $u(t)=(\vec{Q}+v)(t, x-c(t))$. If $v(0) \in B_{\delta}(\mathcal{H})$, then $u$ solves the original equation (1.1) as long as $v(t) \in B_{\delta}(\mathcal{H})$. Meanwhile, the momentum $P(u)=\omega(v, \nabla \vec{Q})+\omega(v, \nabla v) / 2$ is preserved, and so is $\omega(v, J \nabla \vec{Q})$, because of $\dot{c}=A(v)$. Hence if $v(0) \in \mathcal{H}_{\perp} \cap B_{\delta}(\mathcal{H})$, then $v(t)$ remains there as long as $v(t) \in B_{\delta}(\mathcal{H})$.

To see that the solution stays in the neighborhood for $t<0$, expand the conserved energy by $u=(\vec{Q}+v)(x-c)$

$$
E(u)-J(Q)=-\sum_{k \in K} k \lambda_{k+} \lambda_{k-}+\frac{1}{2}\langle\mathcal{L} \gamma \mid \gamma\rangle-C(v),
$$

where the nonlinear energy $C$ is defined by

$$
C(v):=f\left(Q+v_{1}\right)-f(Q)-\left\langle f^{\prime}(Q) \mid v_{1}\right\rangle-\frac{1}{2}\left\langle f^{\prime \prime}(Q) v_{1} \mid v_{1}\right\rangle=o\left(\|v\|_{\mathcal{H}}^{2}\right) .
$$

Suppose that for some $t_{0}<0$

$$
\|v(0)\|_{\mathcal{H}}=\varepsilon \ll \delta, \quad \max _{t_{0} \leq t \leq 0}\|v(t)\|_{\mathcal{H}}<\delta, \quad\left\|v\left(t_{0}\right)\right\|_{\mathcal{H}} \gg \sqrt{\ell} \delta+\varepsilon(\ll \delta) .
$$

Then $|E(u)-J(Q)| \simeq \varepsilon^{2}$. Since $v(t) \in\lceil G\rfloor$ and $G \in \mathcal{G}_{\ell, \delta},(5.8)$ implies that

$$
\langle\mathcal{L} \gamma \mid \gamma\rangle \lesssim \sum_{k} k\left|\lambda_{k+} \lambda_{k-}\right|+E(u)-J(Q) \lesssim \ell \delta^{2}+\varepsilon^{2} \ll \delta^{2}
$$

for $t_{0} \leq t \leq 0$, which together with $v(t) \in \mathcal{H}_{\perp}$ implies

$$
\sum_{k}\left|\lambda_{k+}\left(t_{0}\right)\right|^{2} \simeq\left\|v\left(t_{0}\right)\right\|_{\mathcal{H}}^{2} .
$$

Now consider the nonlinear energy functional

$$
\begin{aligned}
\mathcal{E}(v): & =E(u)-J(Q)+\sum_{k \in K} \frac{k}{2}\left(\lambda_{k+}+\lambda_{k-}\right)^{2} \\
& =\sum_{k \in K} \frac{k}{2}\left(\lambda_{k+}^{2}+\lambda_{k-}^{2}\right)+\frac{1}{2}\langle\mathcal{L} \gamma \mid \gamma\rangle-C(v) .
\end{aligned}
$$

Since $v(t) \in \mathcal{H}_{\perp}$, we have $\mathcal{E}(v) \simeq\|v\|_{\mathcal{H}}^{2}$, and moreover, the equation of $\lambda_{k \pm}$ (see (3.3)) together with conservation of $E(u)$ yields

$$
\frac{d}{d t} \mathcal{E}(v)=\sum_{k} k^{2}\left(\lambda_{k+}^{2}-\lambda_{k-}^{2}\right)+o\left(\|v\|_{\mathcal{H}}^{2}\left|\lambda_{k \pm}\right|\right),
$$

and so

$$
\frac{d}{d t} \mathcal{E}\left(v\left(t_{0}\right)\right) \gtrsim \sum_{k} k^{2} \lambda_{k+}^{2} \simeq \mathcal{E}\left(v\left(t_{0}\right)\right) .
$$

Therefore $\mathcal{E}(v(t))$ cannot increase beyond $O\left(\ell \delta^{2}+\varepsilon^{2}\right)$ as $t<0$ decreases. 
In conclusion, for any $v(0) \in\left\lceil\widetilde{G}_{*}\right\rfloor$ such that $\|v(0)\|_{\mathcal{H}} \ll \delta$, the solution $v(t)=$ $U(t) v(0)$ remains on $\left\lceil\widetilde{G}_{*}\right\rfloor,\|v(t)\|_{\mathcal{H}} \ll \delta$ for all $t<0$ and $u(t)=(\vec{Q}+v)(x-c)$ with $\dot{c}=A(v)$ solves the original equation (1.1) for all $t<0$. Thus we obtain a center-unstable manifold of the original equation with zero total momentum.

More precisely, fix $0<\delta^{\prime} \ll \delta$ and let

$$
\mathcal{M}_{c u, 0}:=\left\{(\vec{Q}+U(t) \varphi)(x-c) \mid \varphi \in\left\lceil\widetilde{G}_{*}\right\rfloor,\|\varphi\|_{\mathcal{H}}<\delta^{\prime}, t<0, c \in \mathbb{R}^{d}\right\} ;
$$

then for any initial data $u(0)=(\vec{Q}+U(t) \varphi)(x-c) \in \mathcal{M}_{c u, 0}$, the solution $u(t)$ of $(1.1)$ is on $\mathcal{M}_{c u, 0}$ for all $t \leq 0$ and $P(u(t))=0$. Moreover, $U(t) \varphi \in\left\lceil\widetilde{G}_{*}\right\rfloor$ and $\|U(t) \varphi\|_{\mathcal{H}} \lesssim \delta^{\prime}$ for all $t \leq 0$. The nonlinear projection

$$
P_{\perp}: \mathcal{H}_{0} \ni u \mapsto v \in \mathcal{H}_{\perp} ; \quad u=(\vec{Q}+v)(x-c)
$$

is uniquely defined in a neighborhood of the translation family of stationary solutions

$$
\mathscr{S}_{0}(Q):=\{\vec{Q}(x-q)\}_{q \in \mathbb{R}^{d}} \subset \mathcal{H}_{0}
$$

by solving the equation

$$
0=\omega(v, J \nabla \vec{Q})=\omega(u(x+c), J \nabla \vec{Q})=\omega(u, J \nabla \vec{Q}(x-c)) .
$$

Indeed it can be solved locally by the implicit function theorem, since if $u=\vec{Q}(x-$ $\left.c_{0}\right)+\psi,\|\psi\|_{\mathcal{H}} \lesssim \delta$, then

$$
\begin{aligned}
\nabla_{c} \omega(u, J \nabla \vec{Q}(x-c)) & =-\omega\left(\vec{Q}\left(x-c_{0}\right)+\psi, J \nabla^{2} \vec{Q}(x-c)\right) \\
& =H(Q)+O\left(\left|c-c_{0}\right|+\delta\right) .
\end{aligned}
$$

Since the mapping $u \mapsto c$ thereby defined is smooth, the map $u \mapsto(v, c)$ is (locally) bi-Lipschitz in the mobile distance from $\mathcal{H}_{0}$ to $\mathcal{H}_{\perp} \oplus \mathbb{R}^{d}$. Since $\mathcal{M}_{c u, 0}$ is mapped onto a 0-neighborhood of $\left\lceil\widetilde{G}_{*}\right\rfloor \oplus \mathbb{R}^{d}$, the codimension of $\mathcal{M}_{c u, 0}$ in $\mathcal{H}_{0}$ is equal to that of $\left\lceil\widetilde{G}_{*}\right\rfloor$ in $\mathcal{H}_{\perp}$, which is $K$.

Thus we have obtained a center-unstable manifold $\mathcal{M}_{c u, 0}$ of $\mathscr{S}_{0}(Q)$ in $\mathcal{H}_{0}$ with codimension $K$. Its time inversion

$$
\overline{\mathcal{M}_{c u, 0}}=: \mathcal{M}_{c s, 0} \subset \mathcal{H}_{0}
$$

is a center-stable manifold of $\mathscr{S}_{0}(Q)$.

5.3. Lorentz extension of the center-stable manifold. Using the Lorentz transform

$$
u(t, x) \mapsto u_{p}:=u\left(\langle p\rangle t+p \cdot x, x+p(\langle p\rangle-1)|p|^{-2} p \cdot x+t p\right) \quad\left(p \in \mathbb{R}^{d}\right),
$$

we can further extend $\mathcal{M}_{c s, 0}$ to a manifold $\mathcal{M}_{c s}$ of codimension $K$ around the soliton manifold $\mathscr{S}(Q)$. Indeed, (1.1) is invariant for any Lorentz transform, while the total energy and momentum are transformed,

$$
E\left(u_{p}\right)=E(u)\langle p\rangle+P(u) \cdot p, \quad P\left(u_{p}\right)=P(u)\langle p\rangle+E(u) p .
$$

Copyright (C) by SIAM. Unauthorized reproduction of this article is prohibited. 
$E^{2}-|P|^{2}$ is invariant, which is positive around $\mathscr{S}(Q)$. Hence there is a unique $p \in \mathbb{R}^{d}$ for each solution $u$ near the traveling waves such that $P\left(u_{p}\right)=0$ and $E\left(u_{p}\right)=$ $\sqrt{E(u)^{2}-|P(u)|^{2}}$.

However, one needs to be more careful because the Lorentz transform mixes space-time and a solution from $M_{c s, 0}$ may not be global in the negative time. Indeed, from $[17,18]$ we know that "half" of the solutions on $\mathcal{M}_{c s, 0}$ (namely, as given by the separating surface $\mathcal{M}_{c u, 0}$ ) blow up in negative time, at least when $\vec{Q}$ is the ground state and $f(u)=|u|^{p+1}, p>1+4 / d$.

The local well-posedness implies that for any $T>0$ there is $\delta>0$ such that for any initial data within distance $\delta$ from $\mathscr{S}_{0}(Q)$, the solution extends at least for times $|t|<T$. The exponential decay of $Q$ implies that for any $\delta>0$ there is $R>0$ such that for any such initial data, the free energy in the exterior region $|x-q|>R$ is less than $O\left(\delta^{2}\right)$ for some $q \in \mathbb{R}^{d}$. The local well-posedness, the conservation of the energy, and the Sobolev inequality imply that every solution with small initial free energy is global, keeping the same size of free energy for all time. Hence the finite speed of propagation of the free Klein-Gordon equation implies that for small $\delta>0$, every solution with free energy $O\left(\delta^{2}\right)$ in $|x-q|>R$ at $t=0$ is extended to the whole exterior cone $|x-q|>R+|t|$ with the same size of the free energy on any time slice of it.

Thus, in conclusion, there is $R>0$ and $\delta(T)>0$ for any $T>0$ such that every solution starting on $\mathcal{M}_{c s, 0}$ and within distance $\delta$ from $\mathscr{S}_{0}(Q)$ is extended to the space-time region

$$
\left\{(t, x) \in \mathbb{R}^{1+d} \mid t>-T \text { or }|x-q|>R+|t|\right\}
$$

for some $q \in \mathbb{R}^{d}$. For any Lorentz transform $L$, there is $T>0$ such that the image of the above region under $L$ contains $\{(t, x) \mid t \geq 0\}$. In other words, the image of any solution on $\mathcal{M}_{c s, 0}$ close to $\mathscr{S}_{0}(Q)$ is extended to a forward global solution. The invariance of the solution set of $\mathcal{M}_{c s, 0}$ for the space and backward time translations is also inherited by the image, because such a translation of the Lorentz transform is the Lorentz transform of another translation. It is also easy to see that this solution remains close to the corresponding traveling wave.

However, it seems difficult to make the above argument uniform with respect to the Lorentz transform: the larger the momentum $p$, the smaller the neighborhood of $\mathscr{S}_{0}(Q)$ needs to be chosen. This is why the resulting manifold is not strictly Lorentz invariant but only within a neighborhood of $\mathscr{S}(Q)$ depending on the Lorentz transform (but the neighborhood can be chosen uniformly for $p$ in compact sets).

Thus we obtain a center-stable manifold $\mathcal{M}_{c s}$ of the soliton manifold $\mathscr{S}(Q)$. $\mathcal{M}_{c s}$ can be identified with the set of forward global solutions starting from it, where each solution is characterized uniquely by the total momentum and its Lorentz transform with 0 -momentum starting from $\mathcal{M}_{c s, 0}$. In this way, ${ }^{2}$ we can define a bi-Lipschitz map from a neighborhood of $\mathscr{S}(Q)$ in $\mathcal{H}$ to a neighborhood of $\mathscr{S}_{0}(Q) \oplus \mathbb{R}^{d}$ in $\mathcal{H}_{0} \oplus \mathbb{R}^{d}$. Since it maps $\mathcal{M}_{c s}$ onto the intersection of $\mathcal{M}_{c s, 0} \oplus \mathbb{R}^{d}$ with a neighborhood of $\mathscr{S}_{0}(Q) \oplus \mathbb{R}^{d}$, the codimension of $\mathcal{M}_{c s}$ in $\mathcal{H}$ is also $K$.

5.4. Solutions off the center-stable manifold. It remains to describe the dynamics of the manifold or, more specifically, the repulsive property of the centerunstable manifold in negative time. For this we need some sort of opposite to Lemma 3.3 .

\footnotetext{
${ }^{2}$ The general solution close to $\mathscr{S}(Q)$ may well blow up in both time directions, but the smaller neighborhood yields the bigger lower bound on the existence time, which is sufficient for the construction of this bi-Lipschitz map.
} 
LEMma 5.2. If $\ell, \delta>0$ satisfy

$$
\delta \ll \ell \underline{k},
$$

then for any two solutions $v^{j}=U(t) v^{j}(0)(j=0,1)$ satisfying

$$
\max \left(\left\|\triangleleft v_{0+}^{\triangleright}(0)\right\|_{E}, \tilde{\mathfrak{m}}_{\delta} \gamma^{\triangleright}(0)\right) \leq \ell\left\|\triangleleft v_{-}^{\triangleright}(0)\right\|_{E},
$$

one has

$$
\max \left(\left\|\triangleleft v_{0+}^{\triangleright}(t)\right\|_{E}, \tilde{\mathfrak{m}}_{\delta} \gamma^{\triangleright}(t)\right) \leq \begin{cases}2 \ell\left\|\triangleleft v_{-}^{\triangleright}(t)\right\|_{E} & (-1 / 2<t \leq 0), \\ \ell\left\|\triangleleft v_{-}^{\triangleright}(t)\right\|_{E} & (-1 \leq t \leq-1 / 2)\end{cases}
$$

and

$$
\left\|\triangleleft v_{-}^{\triangleright}(t)\right\|_{E} \geq \begin{cases}\frac{1}{2} e^{-\underline{k} t / 2}\left\|\triangleleft v_{-}^{\triangleright}(0)\right\|_{E} & (-1 / 2<t \leq 0), \\ e^{-\underline{k} t / 2}\left\|\triangleleft v_{-}^{\triangleright}(0)\right\|_{E} & (-1 \leq t \leq-1 / 2) .\end{cases}
$$

Proof. Let $\widetilde{m}(0):=\left\|\triangleleft v_{-}^{\triangleright}(0)\right\|_{E} \simeq \tilde{\mathfrak{m}}_{\delta} v^{\triangleright}(0)$. Lemma 3.2 implies that

$$
\begin{aligned}
\left\|\triangleleft v_{0+}^{\triangleright}(t)\right\|_{E} & \leq\left(e^{-\kappa t} \ell+C \delta\right) \widetilde{m}(0), \quad\left\|\triangleleft v_{-}^{\triangleright}(t)\right\|_{E} \geq\left(e^{-\underline{k} t}-C \delta\right) \widetilde{m}(0), \\
\tilde{\mathfrak{m}}_{\delta} \gamma^{\triangleright}(t) & \leq(\ell+C \delta) \widetilde{m}(0),
\end{aligned}
$$

for $-1 \leq t \leq 0$. Hence

$$
\begin{aligned}
\max \left(\left\|\triangleleft v_{0+}^{\triangleright}(t)\right\|_{E}, \tilde{\mathfrak{m}}_{\delta} \gamma^{\triangleright}(t)\right) & \leq\left(e^{-\kappa t} \ell+C \delta\right)\left(e^{-\underline{k} t}-C \delta\right)^{-1}\left\|\triangleleft v_{-}^{\triangleright}(t)\right\|_{E} \\
& \leq e^{(\underline{k}-\kappa) t}(\ell+C \delta)\left\|\triangleleft v_{-}^{\triangleright}(t)\right\|_{E},
\end{aligned}
$$

and the conclusion follows from (5.25) as well as $\kappa \ll \underline{k}$.

Let $v^{0}(0) \in B_{\delta}(\mathcal{H}) \cap \mathcal{H}_{\perp} \backslash\left\lceil\widetilde{G}_{*}\right\rfloor$, and

$$
\psi:=P_{\gamma+} v^{0}(0), \quad v^{1}(0):=\psi+\widetilde{G}_{*}(\psi) .
$$

Then we have

$$
\triangleleft v_{\gamma+}^{\triangleright}(0)=0, \quad\left\|\triangleleft v_{0}^{\triangleright}(0)\right\|_{E}^{2} \lesssim\left\|\triangleleft v^{\triangleright}(0)\right\|_{E}^{2} \simeq\left\|\triangleleft v_{-}^{\triangleright}(0)\right\|_{E}^{2} \lesssim \delta^{2} .
$$

Hence we can repeatedly apply the above lemma to deduce that

$$
\left\|\triangleleft v_{-}^{\triangleright}(t)\right\|_{E} \geq \frac{1}{2} e^{-\underline{k} t / 2}\left\|\triangleleft v_{-}^{\triangleright}(0)\right\|_{E}
$$

for all $t<0$. In particular,

$$
\left\|v_{-}^{0}(t)\right\|_{E} \geq\left\|\triangleleft v_{-}^{\triangleright}(t)\right\|_{E}-\left\|v_{-}^{1}(t)\right\|_{E} \gg \delta
$$

for sufficiently large $-t$.

In short, any solution starting from $\mathcal{H}_{\perp} \cap B_{\delta}(\mathcal{H}) \backslash\left\lceil\widetilde{G}_{*}\right\rfloor$ moves out of the neighborhood $B_{\delta}(\mathcal{H})$ for large $-t$. Of course, this is meaningful for the original equation only until the (backward) exiting time, but it implies that any trapped solution in $\mathcal{H}_{\perp}$ within distance $\delta$ must be on the manifold $\left\lceil\widetilde{G}_{*}\right\rfloor$ for large $-t$.

Copyright (c) by SIAM. Unauthorized reproduction of this article is prohibited. 
Combining this with the result in the previous section, we conclude that the local center-unstable manifold $\mathcal{M}_{c u, 0}$ is characterized as the collection of solutions with 0 -momentum which stay close to $\mathscr{S}_{0}(Q)$ for all $-t \geq 0$. By symmetry, $\mathcal{M}_{c s, 0}$ is the collection of solutions with 0 -momentum which stay close to $\mathscr{S}_{0}(Q)$ for all $t \geq 0$.

Let $\widetilde{\mathcal{M}}_{c u, 0}$ be the maximal forward evolution of $\mathcal{M}_{c u, 0}$, and let $\widetilde{\mathcal{M}}_{c s, 0}$ be the maximal backward evolution of $\mathcal{M}_{c s, 0}$. Then $\widetilde{\mathcal{M}}_{c s, 0}$ is the collection of solutions that stay close to $\mathscr{S}_{0}(Q)$ for large $t$, namely, the initial data set for which the solution will be trapped by $\mathscr{S}_{0}(Q)$. We have the same characterization for $\widetilde{\mathcal{M}}_{c u, 0}$ for $t \rightarrow-\infty$. By the Lorentz transform, we can extend them to solutions with nonzero momentum which are trapped by $\mathscr{S}(Q)$ with the same momentum.

6. Regularity of the center-stable manifold. The above construction implies only Lipschitz continuity of the manifold. For the differential structure of $\left\lceil G_{*}\right\rfloor$, we also have to take account of the spatial translation. In the following, we assume that $f$ satisfies $(1.7)$ and $\alpha:=\max (1, p-2)$.

Definition 6.1. Let $Y$ be a Banach space. We say that a function $G: \mathcal{H} \rightarrow Y$ is mobile-differentiable at $\varphi \in \mathcal{H}$ if there is a bounded linear $M: \mathcal{H} \times \mathbb{R}^{d} \rightarrow Y$ such that

$$
\lim _{\varepsilon \rightarrow 0}\left\|G\left(\varphi_{(\varepsilon)}\right)-\left[G\left(\varphi^{0}\right)+\varepsilon M(\psi, q)\right]\right\| / \varepsilon=0,
$$

where $\varphi_{(\varepsilon)}:=(\varphi+\varepsilon \psi)(x+\varepsilon q)$, for any $(\psi, q) \in \mathcal{H} \times \mathbb{R}^{d}$. It is obvious that $M$ is unique. We call $\mathscr{D} G(\varphi):=M$ the mobile derivative of $G$ at $\varphi$.

Let $G^{\prime}(\varphi)$ be the usual derivative in the Frechét sense. Then we have

$$
\mathscr{D} G(\varphi)(\psi, q)=G^{\prime}(\varphi)(\psi+\nabla \varphi \cdot q),
$$

provided that $G$ is differentiable in the $\mathcal{D H}$ topology, but in general, it makes sense only in the subspace $q=0$. Hence the mobile-differentiability is stronger than the differentiability in $\mathcal{H}$ and weaker than that in $\mathcal{D H}$.

If $G \in \mathcal{G}_{\ell, \delta}$ and mobile-differentiable, then

$$
\left\|G\left(\varphi_{(\varepsilon)}\right)-G\left(\varphi^{0}\right)\right\|_{\mathcal{H}} \lesssim \ell \mathfrak{m}_{\phi}\left(\varphi_{(\varepsilon)}, \varphi^{0}\right) \lesssim \ell \varepsilon\left[\|\psi\|_{\mathcal{H}}+|q| \phi_{\delta}\left(\|\varphi\|_{\mathcal{H}}\right)\right],
$$

which implies

$$
\|\mathscr{D} G(\varphi)(\psi, q)\|_{\mathcal{H}} \lesssim \ell\left[\|\psi\|_{\mathcal{H}}+|q| \phi_{\delta}\left(\|\varphi\|_{\mathcal{H}}\right)\right] .
$$

Moreover, we have

$$
\begin{aligned}
G((\varphi+\varepsilon \psi+o(\varepsilon))(x+\varepsilon q+o(\varepsilon)) & =G\left(\varphi_{(\varepsilon)}\right)+o(\varepsilon) \\
& =G(\varphi)+\varepsilon \mathscr{D} G(\varphi)(\psi, q)+o(\varepsilon) .
\end{aligned}
$$

We are going to prove that $G_{*}: \mathcal{H} \rightarrow P_{-} \mathcal{H}$ is "mobile- $C^{1, \alpha "}$ by showing the flow-invariance of the following set of such graphs.

Definition 6.2. For each $\delta, \ell, \Lambda>0$, and $\alpha \in(0,1]$, we define $\mathcal{G}_{\ell, \delta}^{\alpha, \Lambda}$ as the set of all $G \in \mathcal{G}_{\ell, \delta}$ that are mobile differentiable at every $\varphi \in \mathcal{H}$, satisfying

$$
\begin{aligned}
& \left\|\mathscr{D} G\left(\varphi^{0}\right)(\psi, q)-\mathscr{D} G\left(\varphi^{1}\right)\left(\tau_{b}^{*} \psi, q\right)\right\|_{E} \\
& \quad \leq \Lambda\left[\left\|\varphi^{0}-\tau_{b} \varphi^{1}\right\|_{E}+|b| \phi_{\delta}\left(\left\|\varphi^{1}\right\|_{E}\right)\right]^{\alpha, 1}\left[\|\psi\|_{E}+|q| \phi_{\delta}\left(\left\|\varphi^{1}\right\|_{E}\right)\right]
\end{aligned}
$$


for all $\varphi^{0}, \varphi^{1}, \psi \in \mathcal{H}$, and $q, b \in \mathbb{R}^{d}$, where

$$
x^{\alpha, 1}:=|x|^{\alpha}+|x| .
$$

We will prove that $\mathcal{G}_{\ell, \delta}^{\alpha, \Lambda}$ is invariant by the flow, provided that $\delta, \ell$ are small and $\Lambda$ is large. First we investigate the backward evolution of the mobile derivative. Assuming the smallness of $\ell, \delta>0$ as in (3.55) and (3.71), for any $G \in \mathcal{G}_{\ell, \delta}$ and $t>0$, define $G_{t}: \mathcal{H} \rightarrow P_{-} \mathcal{H}$ and $\widehat{G}_{t}: \mathcal{H} \rightarrow \mathcal{H}$ by

$$
G_{t}:=\mathcal{U}(t) G, \quad \widehat{G}_{t}(\varphi):=\varphi_{\geq 0}+G_{t}(\varphi) .
$$

Let $\psi \in \mathcal{H}, q, b \in \mathbb{R}^{d}$, and $t_{0} \in[0,1]$. For small $\varepsilon \in \mathbb{R}$, let

$$
v_{(\varepsilon)}(t):=U\left(t-t_{0}\right) \widehat{G}_{t_{0}}\left(\varphi_{(\varepsilon)}\right), \quad w_{(\varepsilon)}(t, x)=v_{(\varepsilon)}\left(t, x-c_{(\varepsilon)}\right),
$$

where $(w, c)=\left(w_{(\varepsilon)}, c_{(\varepsilon)}\right)$ is the solution of (3.7) with the initial data

$$
w_{(\varepsilon)}\left(t_{0}\right)=(\varphi+\varepsilon \psi)(x-b), \quad c_{(\varepsilon)}\left(t_{0}\right)=b+\varepsilon q .
$$

Since the nonlinear term $(F, B)(w, c)$ in $(3.7)$ is $C^{1}$ from $\operatorname{Str} \times L_{t}^{\infty}$ to $L_{t}^{1} \mathcal{H} \times L_{t}^{\infty}$, with a small factor on a short time interval $(0, T)$, it is straightforward by the iteration argument that $(w, c)$ is differentiable in $\operatorname{Str} \times L_{t}^{\infty}$ at $\varepsilon=0$, with the derivative

$$
\begin{aligned}
(z, g) & :=\lim _{\varepsilon \rightarrow 0} \frac{\left(w_{(\varepsilon)}, c_{(\varepsilon)}\right)-\left(w_{(0)}, c_{(0)}\right)}{\varepsilon}, \\
\|z\|_{\operatorname{Str}(0,1)}+\|g\|_{L^{\infty}(0,1)} & \lesssim\left\|z\left(t_{0}\right)\right\|_{\mathcal{H}}+\left|g\left(t_{0}\right)\right| .
\end{aligned}
$$

Let $\eta:=z\left(t, x+c_{(0)}(t)\right)$ and $\widehat{F}=F-\left(0, f^{\prime}\left(Q_{c_{(0)}}\right) w_{1}\right)$. Then

$$
\begin{aligned}
\dot{\eta} & =J \mathcal{L} \eta+\left(0, g \cdot \nabla f^{\prime}(Q) v_{1}\right)+B(w, c) \cdot \nabla \eta+\tau_{c}^{*} \partial_{(w, c)} \widehat{F}(w, c) \cdot(z, g), \\
\eta\left(t_{0}\right) & =P_{\geq 0}(\psi+q \cdot \nabla \varphi)-q \cdot \nabla \widehat{G}_{t_{0}}(\varphi)+\mathscr{D} G_{t_{0}}(\varphi)(\psi, q), \quad g\left(t_{0}\right)=q,
\end{aligned}
$$

where the subscript (0) is omitted. Mobile-differentiating the identities

$$
P_{-} v_{(\varepsilon)}\left(t_{0}\right)=G_{t_{0}}\left(\varphi_{(\varepsilon)}\right), \quad P_{-} v_{(\varepsilon)}(t)=G_{t}\left(v_{(\varepsilon)}\left(t_{0}\right)\right)
$$

yields

$$
\begin{aligned}
P_{-}\left[\eta\left(t_{0}\right)+q \cdot \nabla \widehat{G}_{t_{0}}(\varphi)\right] & =\mathscr{D} G_{t_{0}}(\varphi)(\psi, q), \\
P_{-}[\eta(t)+g(t) \cdot \nabla v(t)] & =\mathscr{D} G_{t}(v(t))(\eta(t), g(t)) .
\end{aligned}
$$

Since $G(\varphi)=G\left(P_{\geq 0} \varphi\right)$ and

$$
P_{\geq 0} \varphi_{(\varepsilon)}=P_{\geq 0}\left[\left(\varphi_{\geq 0}+\varepsilon \psi_{\geq 0}\right)(x+\varepsilon q)+\varepsilon q \cdot \nabla \varphi_{-}\right]+o(\varepsilon),
$$

we have

$$
\mathscr{D} G(\varphi)(\psi, q)=\mathscr{D} G\left(\varphi_{\geq 0}\right)\left(\psi_{\geq 0}+q \cdot P_{\geq 0} \nabla \varphi_{-}, q\right) .
$$

LEMma 6.3. Let $\ell, \delta, \Lambda>0$ satisfy (3.55), (3.71), and

$$
\Lambda \gg \ell / \delta \text {. }
$$

Then for any $G \in \mathcal{G}_{\ell, \delta}^{\alpha, \Lambda}$ and any $t_{0} \in[1 / 2,1], \mathcal{U}(t) G \in \mathcal{G}_{\ell, \delta}^{\alpha, \Lambda}$. 
Proof. First, (6.4) is enough to have (6.6) in the case where

$$
\ell / \Lambda \ll\left\|\varphi^{0}-\tau_{b} \varphi^{1}\right\|_{E}+|b| \phi_{\delta}\left(\varphi^{1}\right) .
$$

Hence we may assume

$$
\mathfrak{m}_{\phi} \varphi^{\triangleright} \leq\left\|\varphi^{0}-\tau_{b} \varphi^{1}\right\|_{E}+|b| \phi_{\delta}\left(\varphi^{1}\right) \lesssim \ell / \Lambda \ll \delta .
$$

Therefore we have either $\left\|\varphi^{0}\right\|_{\mathcal{H}} \simeq\left\|\varphi^{1}\right\| \gg \delta$ or $\left\|\varphi^{0}\right\|_{\mathcal{H}}+\left\|\varphi^{1}\right\|_{\mathcal{H}} \lesssim \delta$.

Next we investigate evolution of the difference of mobile-derivatives. For any $\varphi^{0}, \varphi^{1}, \psi \in \mathcal{H}$, and $q, b \in \mathbb{R}^{d}$, let

$$
\begin{aligned}
v_{(\varepsilon)}^{0}\left(t_{0}\right) & :=\widehat{G}_{t_{0}}\left(\tau_{\varepsilon q}^{*}\left(\varphi^{0}+\varepsilon \psi\right)\right), & v_{(\varepsilon)}^{1}\left(t_{0}\right) & :=\widehat{G}_{t_{0}}\left(\tau_{\varepsilon q}^{*}\left(\varphi^{1}+\varepsilon \tau_{b}^{*} \psi\right)\right), \\
v_{(\varepsilon)}^{j}(t) & :=U\left(t-t_{0}\right) v_{(\varepsilon)}^{j}\left(t_{0}\right), & w_{(\varepsilon)}^{j}(t) & :=\tau_{c_{(\varepsilon)}^{j}(t)} v_{(\varepsilon)}^{j}(t)
\end{aligned}
$$

for $j=0,1$, where $c_{(\varepsilon)}^{j}$ is the solution of

$$
\dot{c}_{(\varepsilon)}^{j}=B\left(w_{(\varepsilon)}^{j}, c_{(\varepsilon)}^{j}\right), \quad c_{(\varepsilon)}^{0}\left(t_{0}\right)=\varepsilon q, \quad c_{(\varepsilon)}^{1}\left(t_{0}\right)=b+\varepsilon q .
$$

Let $\left(z^{j}, g^{j}\right)$ be the derivative at $\varepsilon=0$ of $\left(w_{(\varepsilon)}^{j}, c_{(\varepsilon)}^{j}\right)$ :

$$
z^{j}=\lim _{\varepsilon \rightarrow 0} \frac{w_{(\varepsilon)}^{j}-w_{(0)}^{j}}{\varepsilon}, \quad g^{j}=\lim _{\varepsilon \rightarrow 0} \frac{c_{(\varepsilon)}^{j}-c_{(0)}^{j}}{\varepsilon} .
$$

Henceforth the subscript (0) will be omitted. The initial values are

$$
\begin{aligned}
w^{0}\left(t_{0}\right) & =\widehat{G}_{t_{0}}\left(\varphi^{0}\right), \quad c^{0}\left(t_{0}\right)=0, \quad w^{1}\left(t_{0}\right)=\tau_{b} \widehat{G}_{t_{0}}\left(\varphi^{1}\right), \quad c^{1}\left(t_{0}\right)=b, \\
z^{0}\left(t_{0}\right) & =P_{\geq 0} \psi+q \cdot \check{G}_{t_{0}}\left(\varphi^{0}\right)+\mathscr{D} G_{t_{0}}\left(\varphi^{0}\right)(\psi, q), \quad g^{0}\left(t_{0}\right)=q, \\
z^{1}\left(t_{0}\right) & =P_{\geq 0}^{b} \psi+\tau_{b}\left[q \cdot \check{G}_{t_{0}}\left(\varphi^{1}\right)+\mathscr{D} G_{t_{0}}\left(\varphi^{1}\right)\left(\tau_{b}^{*} \psi, q\right)\right], \quad g^{1}\left(t_{0}\right)=q,
\end{aligned}
$$

where $P_{*}^{b}:=\tau_{b} P_{*} \tau_{b}^{*}$, and $\check{G}_{t}: \mathcal{H} \rightarrow \mathcal{H}^{d}$ is defined by

$$
\check{G}_{t}(\varphi)=\nabla P_{-} \varphi-P_{-} \nabla \varphi-\nabla G_{t}(\varphi) .
$$

Let $\eta^{j}(t, x)=z^{j}\left(t, x+c^{j}(t)\right)$, then we have

$$
\begin{aligned}
P_{-}\left[\eta^{0}\left(t_{0}\right)+q \cdot \nabla \widehat{G}_{t_{0}}\left(\varphi^{0}\right)\right] & =\mathscr{D} G_{t_{0}}\left(\varphi^{0}\right)(\psi, q), \\
P_{-}\left[\eta^{1}\left(t_{0}\right)+q \cdot \nabla \widehat{G}_{t_{0}}\left(\varphi^{1}\right)\right] & =\mathscr{D} G_{t_{0}}\left(\varphi^{1}\right)\left(\tau_{b}^{*} \psi, q\right), \\
P_{-}\left[\eta^{j}(t)+g^{j}(t) \cdot \nabla v^{j}(t)\right] & =\mathscr{D} G_{t}\left(v^{j}(t)\right)\left(\eta^{j}(t), g^{j}(t)\right) .
\end{aligned}
$$

Thus we obtain

$$
\begin{aligned}
\mathscr{D} G_{t_{0}} & \left(\varphi^{0}\right)(\psi, q)-\mathscr{D} G_{t_{0}}\left(\varphi^{1}\right)\left(\tau_{b}^{*} \psi, q\right) \\
= & \triangleleft\left[\eta_{-}^{\triangleright}\left(t_{0}\right)+q \cdot P_{-} \nabla \widehat{G}_{t_{0}}\left(\varphi^{\triangleright}\right)\right] \\
= & e^{J \mathcal{L} t_{0}} \triangleleft \mathscr{D} G_{0}\left(v^{\triangleright}(0)\right)\left(\eta^{\triangleright}(0), g^{\triangleright}(0)\right) \\
& -e^{J \mathcal{L} t_{0}} \triangleleft g^{\triangleright}(0) \cdot P_{-} \nabla v^{\triangleright}(0) \\
& +\triangleleft\left[\eta_{-}^{\triangleright}\left(t_{0}\right)-e^{J L_{0} t_{0}} \eta_{-}^{\triangleright}(0)\right]+q \cdot \triangleleft P-\nabla \widehat{G}_{t_{0}}\left(\varphi^{\triangleright}\right) .
\end{aligned}
$$

Copyright (c) by SIAM. Unauthorized reproduction of this article is prohibited. 
The first term on the right of (6.26) can be rewritten by using (6.16)

$$
\begin{aligned}
\triangleleft \mathscr{D} G_{0}\left(v^{\triangleright}\right)\left(\eta^{\triangleright}, g^{\triangleright}\right)= & \triangleleft \mathscr{D} G_{0}\left(v_{\geq 0}^{0}\right)\left(\eta_{\geq 0}^{0}+\mathcal{R} v^{0}, g^{0}\right) \\
& -\triangleleft \mathscr{D} G_{0}\left(v_{\geq 0}^{1}\right)\left(\eta_{\geq 0}^{1}+\mathcal{R} v^{1}, g^{0}\right)+\mathscr{D} G_{0}\left(v^{1}\right)\left(\eta^{1}, \triangleleft g^{\triangleright}\right),
\end{aligned}
$$

where all functions are evaluated at $t=0$, and the operator $\mathcal{R}$ is defined by

$$
\mathcal{R}:=g^{0} \cdot P_{\geq 0} \nabla P_{-} .
$$

We say that a component in (6.26) is negligible if its norm in $E$ is much smaller than the right-hand side of (6.6). So is the last term in (6.26), since $\Lambda \gg 1$ and

$$
\left\|\triangleleft P_{-} \nabla \widehat{G}_{t_{0}}\left(\varphi^{\triangleright}\right)\right\|_{E} \lesssim\left\|D^{-1} \triangleleft \widehat{G}_{t_{0}}\left(\varphi^{\triangleright}\right)\right\|_{\mathcal{H}} \lesssim \tilde{\mathfrak{m}}_{\delta} \varphi^{\triangleright} \lesssim\left\|\varphi^{0}-\tau_{b} \varphi^{1}\right\|_{E} .
$$

In order to estimate the other terms, we prepare rough bounds on the unknowns. Lemma 3.1 together with $G \in \mathcal{G}_{\ell, \delta}$ implies that

$$
\begin{aligned}
\left\|v^{j}\right\|_{\operatorname{Str}(0,1)} \lesssim\left\|\varphi^{j}\right\|_{E}, \quad\left\|v_{\geq 0}^{j}(0)\right\|_{E} & \leq(1+C \kappa+C \delta)\left\|v_{\geq 0}\left(t_{0}\right)\right\|_{E} \\
& \leq(1+C \kappa+C \delta+C \ell)\left\|\varphi_{\geq 0}^{j}\right\|_{E} .
\end{aligned}
$$

The estimates in (3.1) together with $G \in \mathcal{G}_{\ell, \delta}$ imply

$$
\begin{aligned}
\left\|\triangleleft w^{\triangleright}\right\|_{\operatorname{Str}(0,1)}+\left\|\triangleleft c^{\triangleright}\right\|_{L^{\infty}(0,1)} \lesssim & \left\|\triangleleft w^{\triangleright}\left(t_{0}\right)\right\|_{\mathcal{H}}+\left|\triangleleft c^{\triangleright}\left(t_{0}\right)\right| \\
\lesssim & \left\|\varphi_{\geq 0}^{0}-\tau_{b} \varphi_{\geq 0}^{1}\right\|_{E}+|b| \ell\left\|\varphi^{1}\right\|_{E} \\
& +\ell\left(\tilde{\mathfrak{m}}_{\delta} \varphi_{\geq 0}^{\triangleright}+|b| \phi_{\delta}\left(\left\|\varphi^{1}\right\|_{E}\right)\right)+|b| \\
\lesssim & \left\|\varphi^{0}-\tau_{b} \varphi^{1}\right\|_{E}+|b| \phi_{\delta}\left(\left\|\varphi^{1}\right\|_{E}\right) .
\end{aligned}
$$

The equation for each $\left(z^{j}, g^{j}\right)$ is given as follows:

$$
\begin{aligned}
\dot{g} & =B^{\prime}, \quad \dot{z}=F^{\prime}, \\
\chi_{\delta}^{\prime} & :=2 \delta^{-2} \chi^{\prime}\left(\|w\|_{\mathcal{H}}^{2} / \delta^{2}\right)\langle w, z\rangle_{\mathcal{H}}, \quad I:=\left(H(Q)-\left\langle\nabla^{2} Q_{c} \mid w_{1}\right\rangle\right)^{-1}, \\
B^{\prime} & :=\chi_{\delta}^{\prime} I \frac{\omega(w, \nabla w)}{2}+I\left(\left\langle\nabla Q_{c} \mid z_{1}\right\rangle-g\left\langle\nabla^{2} Q_{c} \mid w_{1}\right\rangle\right) B+\chi_{\delta} I \omega(z, \nabla w), \\
F^{\prime} & :=\left(\begin{array}{c}
B^{\prime} \cdot \nabla Q_{c}+g \cdot \nabla^{2} Q_{c} \cdot B \\
f^{\prime}\left(Q_{c}\right) z_{1}-g \cdot \nabla f^{\prime}\left(Q_{c}\right) w_{1}+N^{\prime}
\end{array}\right), \\
N^{\prime} & :=\chi_{\delta}^{\prime} N_{c}+\chi_{\delta}\left[\left(f^{\prime}\left(Q_{c}+w_{1}\right)-f^{\prime}\left(Q_{c}\right)\right)\left(z_{1}-g \cdot \nabla Q_{c}\right)+g \cdot \nabla f^{\prime}\left(Q_{c}\right) w_{1}\right],
\end{aligned}
$$

where the superscript $j$ and the dependence on $(w, c)$ are omitted. Using the estimate $\|w\|_{\operatorname{Str}(-1,1)} \lesssim\left\|w\left(t_{0}\right)\right\|_{\mathcal{H}}$, we obtain in the same way as for (3.1),

$$
\begin{aligned}
\left|\chi_{\delta}^{\prime}\right| & \lesssim\|z\|_{\mathcal{H}} / \delta, \quad|B| \lesssim \delta^{2}, \quad\left|B^{\prime}\right| \lesssim \delta\|z\|_{\mathcal{H}}+|g|\left(\|w(0)\|_{\mathcal{H}} \wedge \delta\right)^{3} \\
\left\|F^{\prime}\right\|_{L^{1} \mathcal{H}(-1,1)} & \lesssim\left[T+\left(\left\|w\left(t_{0}\right)\right\|_{\mathcal{H}} \wedge \delta\right)\right]\left[\|z\|_{\operatorname{Str}}+\|g\|_{L^{\infty}}\right]+T\left\|w\left(t_{0}\right)\right\|_{\mathcal{H}}\|g\|_{L^{\infty}} .
\end{aligned}
$$

In the case $\left\|\varphi^{0}\right\|_{\mathcal{H}} \simeq\left\|\varphi^{1}\right\|_{\mathcal{H}} \gg \delta$, we have $g^{j} \equiv q$, and so by the Strichartz estimate,

$$
\left\|z^{j}\right\|_{\text {Str }} \lesssim\left\|z^{j}\left(t_{0}\right)\right\|_{\mathcal{H}}+|q|\left\|w^{j}\left(t_{0}\right)\right\|_{\mathcal{H}}
$$

In the other case $\left\|\varphi^{\triangleright}\right\|_{\mathcal{H}} \lesssim \delta$,

$$
\left\|z^{j}\right\|_{\operatorname{Str}}+\left\|g^{j}\right\|_{L^{\infty}} \lesssim\left\|z^{j}\left(t_{0}\right)\right\|_{\mathcal{H}}+\left|g^{j}\left(t_{0}\right)\right| \text {. }
$$

Copyright $@$ by SIAM. Unauthorized reproduction of this article is prohibited. 
In both cases, (6.4) implies

$$
\begin{aligned}
& \left\|z^{j}\right\|_{\operatorname{Str}(0,1)}+\left\|g^{j}\right\|_{L^{\infty}(0,1)} \\
& \quad \lesssim\|\psi\|_{\mathcal{H}}+|q| \ell\left\|\varphi^{j}\right\|_{\mathcal{H}}+\ell\left[\|\psi\|_{\mathcal{H}}+|q| \phi_{\delta}\left(\left\|\varphi^{j}\right\|_{E}\right)\right]+|q| \\
& \quad \lesssim\|\psi\|_{E}+|q| \phi_{\delta}\left(\left\|\varphi^{j}\right\|_{E}\right) .
\end{aligned}
$$

For the difference estimate, we consider the two cases separately. If $\left\|\varphi^{0}\right\|_{\mathcal{H}} \simeq$ $\left\|\varphi^{1}\right\|_{\mathcal{H}} \gg \delta$, then $g^{j} \equiv q$ and

$$
\triangleleft \dot{z}=J \mathcal{D} \triangleleft z+\left(0, \triangleleft f^{\prime}\left(Q_{c^{\triangleright}}\right) z_{1}^{\triangleright}-q \cdot \nabla f^{\prime}(Q) \triangleleft w_{1}^{\triangleright}-q \cdot \triangleleft \nabla f^{\prime}\left(Q_{c^{\triangleright}}\right) w_{1}^{1}\right),
$$

and so

$$
\left\|\triangleleft z^{\triangleright}\right\|_{\operatorname{Str}(0,1)} \lesssim\left\|\triangleleft z^{\triangleright}\left(t_{0}\right)\right\|+|b|\left\|z^{\triangleright}\right\|_{\operatorname{Str}}+|q|\left\|\triangleleft w^{\triangleright}\right\|_{\operatorname{Str}}+|b|^{\alpha}|q|\left\|w^{\triangleright}\right\|_{\operatorname{Str}},
$$

where the last term comes from the last one of (6.37). Inserting (6.31) and (6.36), we obtain

$$
\begin{aligned}
& \left\|\triangleleft\left(z^{\triangleright}, g^{\triangleright}\right)\right\|_{\operatorname{Str} \times L^{\infty}(0,1)} \\
& \quad \lesssim\left[\left\|\varphi^{0}-\tau_{b} \varphi^{1}\right\|_{E}+|b| \phi_{\delta}\left(\left\|\varphi^{1}\right\|_{E}\right)\right]^{\alpha, 1}\left[\|\psi\|+|q| \phi_{\delta}\left(\left\|\varphi^{1}\right\|_{E}\right)\right] .
\end{aligned}
$$

In the nonlinear case $\left\|\varphi^{\triangleright}\right\|_{\mathcal{H}} \lesssim \delta$, we have

$$
\begin{aligned}
\left|\triangleleft \chi_{\delta}^{\prime}\right| & \lesssim \delta^{-2}\left(\left\|\triangleleft w^{\triangleright}\right\|_{\mathcal{H}}\left\|z^{\triangleright}\right\|_{\mathcal{H}}+\left\|w^{\triangleright}\right\|_{\mathcal{H}}\left\|_{\triangleleft z^{\triangleright}}\right\|_{\mathcal{H}}\right), \\
\left|\triangleleft B^{\prime}\right| \lesssim & \lesssim \triangleleft\left(w^{\triangleright}, c^{\triangleright}\right)\left\|_{\mathcal{H} \times \mathbb{R}^{d}}\right\|\left(z^{\triangleright}, g^{\triangleright}\right)\left\|_{\mathcal{H} \times \mathbb{R}^{d}}+\right\| w^{\triangleright}\left\|_{\mathcal{H}}\right\| \triangleleft\left(z^{\triangleright}, g^{\triangleright}\right) \|_{\mathcal{H} \times \mathbb{R}^{d}}, \\
\left\|\triangleleft F^{\prime}\right\|_{L^{1} \mathcal{H}} \lesssim T\left[\left\|\triangleleft z^{\triangleright}\right\|_{\operatorname{Str}}+\delta\left\|\triangleleft c^{\triangleright}\right\|_{L^{\infty}}^{\alpha}\|g\|_{L^{\infty}}\right]+\left\|w^{\triangleright}\right\|_{\operatorname{Str}}\left\|\triangleleft\left(z^{\triangleright}, g^{\triangleright}\right)\right\|_{\operatorname{Str} \times L^{\infty}} & +\left\|\triangleleft\left(w^{\triangleright}, c^{\triangleright}\right)\right\|_{\operatorname{Str} \times L^{\infty}}\left\|\left(z^{\triangleright}, g^{\triangleright}\right)\right\|_{\operatorname{Str} \times L^{\infty}},
\end{aligned}
$$

where the term with $\alpha$ power comes from the same term as in the linear case, i.e., $\left(1-\chi_{\delta}\right) q \cdot \triangleleft \nabla f^{\prime}\left(Q_{c^{\triangleright}}\right) w_{1}^{1}$. Hence

$$
\left\|\triangleleft\left(z^{\triangleright}, g^{\triangleright}\right)\right\|_{\operatorname{Str} \times L^{\infty}(0,1)} \lesssim\left\|\triangleleft z^{\triangleright}\left(t_{0}\right)\right\|_{\mathcal{H}}+\left[\left\|\triangleleft w^{\triangleright}\right\|_{\operatorname{Str}}+\left\|\triangleleft c^{\triangleright}\right\|_{L^{\infty}}^{\alpha, 1}\right]\left\|\left(z^{\triangleright}, g^{\triangleright}\right)\right\|_{\operatorname{Str} \times L^{\infty}},
$$

which, together with (6.31) and (6.36), leads to the same bound (6.39) as in the linear case.

For the penultimate term in (6.26), we obtain from the equation of $\eta^{j}(6.12)$ in the same way as above,

$$
\begin{aligned}
\| \triangleleft P_{-} & {\left[\eta^{\triangleright}\left(t_{0}\right)-e^{J \mathcal{L} t_{0}} \eta^{\triangleright}(0)\right] \|_{E} } \\
\lesssim & \left\|\triangleleft g^{\triangleright}\right\|_{L_{t}^{\infty}}\left\|v^{\triangleright}\right\|_{\operatorname{Str}}+\left\|g^{\triangleright}\right\|_{L_{t}^{\infty}}\left\|\tilde{\mathfrak{m}}_{\delta} v^{\triangleright}\right\|_{L_{t}^{\infty}} \\
\quad & +\delta\left\|\triangleleft\left(z^{\triangleright}, g^{\triangleright}\right)\right\|_{\operatorname{Str} \times L_{t}^{\infty}}+\left\|\triangleleft\left(w^{\triangleright}, c^{\triangleright}\right)\right\|_{\operatorname{Str} \times L_{t}^{\infty}}\left\|\left(z^{\triangleright}, g^{\triangleright}\right)\right\|_{\operatorname{Str} \times L_{t}^{\infty}},
\end{aligned}
$$

where we do not get the term with $\alpha$-power, since the potential term is frozen in the $\eta$ equation. Using (6.30)-(6.36) and Lemma 3.2, we can easily observe that the above is negligible because $\Lambda \gg 1$. Here again we have used that either $\left\|\varphi^{\triangleright}\right\|_{\mathcal{H}} \lesssim \delta$ or $g^{j} \equiv q$, which will be tacitly utilized in the following, too. Hence the second term on the right of (6.26) is also negligible by (6.30) and (6.39).

For the remaining and leading term of (6.26), we have

$$
\begin{aligned}
\| e^{J \mathcal{L} t_{0}} & \triangleleft \mathscr{D} G_{0}\left(v^{\triangleright}\right)\left(\eta^{\triangleright}, g^{\triangleright}\right) \|_{E} \\
\leq & e^{-\underline{k} t_{0}} \Lambda\left[\left\|v_{\geq 0}^{0}-\tau_{\triangleleft c^{\triangleright}} v_{\geq 0}^{1}\right\|_{E}+\left|\triangleleft c^{\triangleright}\right| \phi_{\delta}\left(\left\|v_{\geq 0}^{1}\right\|_{E}\right)\right]^{\alpha, 1} \\
& \times\left[\left\|\eta_{\geq 0}^{0}+\mathcal{R} v^{0}\right\|_{E}+\left|g^{0}\right| \phi_{\delta}\left(\left\|v_{\geq 0}^{1}\right\|_{E}\right)\right] \\
& +C \ell\left|\triangleleft g^{\triangleright}\right| \phi_{\delta}\left(\left\|v_{\geq 0}^{1}\right\|_{E}\right)+C \ell\left\|\tau_{\triangleleft c^{\triangleright}}^{*}\left[\eta_{\geq 0}^{0}+\mathcal{R} v^{0}\right]-\left[\eta_{\geq 0}^{1}+\mathcal{R} v^{1}\right]\right\|_{E},
\end{aligned}
$$

Copyright (c) by SIAM. Unauthorized reproduction of this article is prohibited. 
where $t=0$. The penultimate term in (6.43) is negligible thanks to $\ell \ll \Lambda,(6.30)$ and (6.39). The last term in (6.43) is dominated by

$$
\begin{aligned}
& \left\|\tau_{\triangleleft c^{\triangleright}}^{*} \eta^{0}-\eta^{1}\right\|_{\mathcal{H}}+\left\|\left[P_{-}, \tau_{\triangleleft \triangle^{\triangleright}}^{*}\right] \eta^{0}\right\|_{\mathcal{H}}+\left|\triangleleft c^{\triangleright}\right|\left\|\mathcal{R} v^{0}\right\|_{\mathcal{H}}+\left\|\triangleleft \mathcal{R} v^{\triangleright}\right\|_{\mathcal{H}} \\
& \lesssim\left\|\triangleleft z^{\triangleright}\right\|_{\mathcal{H}}+\left|\triangleleft c^{\triangleright}\right|\left\|z^{0}\right\|_{\mathcal{H}}+\left|\triangleleft c ^ { \triangleright } \left\|g^{0}\left|\left\|v^{0}\right\|_{\mathcal{H}}+\right| g^{0} \mid \tilde{\mathfrak{m}}_{\delta} v^{\triangleright} .\right.\right.
\end{aligned}
$$

Hence it is also negligible by using the estimates (6.30)-(6.39) and $\ell \ll \Lambda$.

It remains to deal with the leading term of (6.43), for which we need more precise estimates, employing the time decay of $e^{-\underline{k} t_{0}}$. First we consider the linearized case $\left\|\varphi^{0}\right\|_{\mathcal{H}} \simeq\left\|\varphi^{1}\right\|_{\mathcal{H}} \gg \delta$, using the equations

$$
\begin{aligned}
v^{j}(0) & =e^{-J \mathcal{L} t_{0}} \widehat{G}_{t_{0}}\left(\varphi^{j}\right), \quad c^{0}(t)=0, \quad c^{1}(t)=b, \\
\dot{\eta}^{j} & =J \mathcal{L} \eta^{j}+\left(0, q \cdot \nabla f^{\prime}(Q) v_{1}^{j}\right), \quad g^{j}(t)=q .
\end{aligned}
$$

The first component on the right of (6.43) is estimated by

$$
\begin{aligned}
& \left\|v_{\geq 0}^{0}-\tau_{\triangleleft \triangleleft^{\triangleright}} v_{\geq 0}^{1}\right\|_{E} \\
& \quad \leq\left\|e^{-J \mathcal{L} t_{0}} P_{\geq 0}\left(\varphi^{0}-\tau_{b} \varphi^{1}\right)\right\|_{E}+\left\|e^{-J \mathcal{L} t_{0}}\left[P_{-}, \tau_{b}\right] \varphi^{1}\right\|_{E}+\left\|\left[e^{-J \mathcal{L} t_{0}}, \tau_{b}\right] \varphi_{\geq 0}^{1}\right\|_{E} \\
& \quad \leq e^{\kappa}\left\|\varphi^{0}-\tau_{b} \varphi^{1}\right\|_{E}+C|b|\left\|\varphi^{1}\right\|_{E}
\end{aligned}
$$

and the third component by

$$
\left\|\eta_{\geq 0}^{0}+\mathcal{R} v^{0}\right\|_{E} \leq\left\|e^{-J \mathcal{L} t_{0}} \eta_{\geq 0}^{0}\left(t_{0}\right)\right\|_{E}+C\left|q\|\| \varphi^{0}\left\|_{E} \leq e^{\kappa}\right\| \psi\left\|_{E}+C|q|\right\| \varphi^{0} \|_{E} .\right.
$$

Since $\underline{k} t_{0} \gg \kappa$ and $\phi_{\delta}\left(\left\|\varphi^{j}\right\|_{E}\right) \gg\left\|\varphi^{j}\right\|_{E}$, we see that the leading term of (6.43) is smaller than

$$
\frac{1}{2} \Lambda\left[\left\|\varphi^{0}-\tau_{b} \varphi^{1}\right\|_{E}+|b| \phi_{\delta}\left(\left\|\varphi^{1}\right\|_{E}\right)\right]\left[\|\psi\|_{E}+|q| \phi_{\delta}\left(\left\|\varphi^{1}\right\|_{E}\right)\right] .
$$

Hence the other terms, which have been shown to be negligible, are absorbed into the remaining half of (6.6). Thus the linearized case $\left\|\varphi^{\triangleright}\right\| \gg \delta$ is done.

It remains to consider the nonlinear case $\left\|\varphi^{\triangleright}\right\| \lesssim \delta$. Let

$$
\xi:=v^{0}-\tau_{\triangleleft c^{\triangleright}} v^{1}=\tau_{c^{0}}^{*} \triangleleft w^{\triangleright},
$$

then we have

$$
\begin{aligned}
v_{\geq 0}^{0}-\tau_{\triangleleft c^{\triangleright}} v_{\geq 0}^{1} & =\xi_{\geq 0}+\left[P_{-}, \tau_{\triangleleft c^{\triangleright}}\right] v^{1}, \\
\dot{\xi} & =J \mathcal{L} \xi+B\left(w^{0}, c^{0}\right) \cdot \nabla \xi+\tau_{c^{0}}^{*}\left[\left(0, \triangleleft f^{\prime}\left(Q_{c^{\triangleright}}\right) w_{1}^{1}\right)+\triangleleft \widehat{F}\left(w^{\triangleright}, c^{\triangleright}\right)\right] .
\end{aligned}
$$

The commutator term is negligible thanks to (6.30), (6.31), and $\left\|\varphi^{1}\right\|_{\mathcal{H}} \lesssim \delta$. For the discrete spectral part of $\xi_{\geq 0}$, we have

$$
\begin{gathered}
\left\|P_{0+}\left[\xi-e^{-J \mathcal{L} t_{0}} \xi\left(t_{0}\right)\right]\right\|_{E} \lesssim \delta\left\|\triangleleft\left(w^{\triangleright}, c^{\triangleright}\right)\right\|_{\operatorname{Str} \times L_{t}^{\infty}(0,1)}, \\
\left\|e^{-J \mathcal{L} t_{0}} \xi_{0+}\left(t_{0}\right)\right\|_{E} \leq e^{\kappa}\left\|P_{0+} \xi\left(t_{0}\right)\right\|_{E},
\end{gathered}
$$

and for the continuous spectral part,

$$
\begin{aligned}
\partial_{t}\left\langle\mathcal{L} \xi \mid P_{\gamma} \xi\right\rangle / 2= & B\left(w^{0}, c^{0}\right)\left[\left\langle f^{\prime}(Q) \xi_{1} \mid \nabla \xi_{1}\right\rangle-\left\langle\mathcal{L} \xi \mid P_{d} \nabla \xi\right\rangle\right] \\
& +\left\langle\mathcal{L} \xi \mid P_{\gamma} \tau_{c^{0}}^{*}\left[\left(0, \triangleleft f^{\prime}\left(Q_{c^{\triangleright}}\right) w_{1}^{1}\right)+\triangleleft \widehat{F}\left(w^{\triangleright}, c^{\triangleright}\right)\right]\right\rangle \\
\lesssim & \delta^{2}\|\xi\|_{E}^{2}+\|\xi\|_{E} \delta\left\|\triangleleft\left(w^{\triangleright}, c^{\triangleright}\right)\right\|_{\operatorname{Str} \times L_{t}^{\infty}(0,1)} .
\end{aligned}
$$

Copyright (c) by SIAM. Unauthorized reproduction of this article is prohibited. 
Thus we deduce

$$
\begin{aligned}
\left\|\xi_{\geq 0}(0)\right\|_{E} & \leq e^{\kappa}\left\|\xi_{\geq 0}\left(t_{0}\right)\right\|_{E}+C \sqrt{\delta}\left\|\triangleleft\left(w^{\triangleright}, c^{\triangleright}\right)\right\|_{\operatorname{Str} \times L_{t}^{\infty}(0,1)} \\
& \leq(1+C \kappa+C \sqrt{\delta})\left[\left\|\varphi^{0}-\tau_{b} \varphi^{1}\right\|_{E}+|b| \phi_{\delta}\left(\left\|\varphi^{1}\right\|_{E}\right)\right] .
\end{aligned}
$$

Similarly for the $\eta^{0}$ component, (6.12) implies

$$
\begin{aligned}
\left\|\eta^{0}\left(t_{0}\right)\right\|_{E} \leq & \|\psi\|_{E}+C|q|\left\|\varphi^{0}\right\|_{E}+C \ell\left(\|\psi\|_{E}+|q|\right), \\
\left\|P_{0+}\left[\eta^{0}(0)-e^{-J \mathcal{L} t_{0}} \eta^{0}\left(t_{0}\right)\right]\right\|_{E} \lesssim & \delta\left\|\left(z^{0}, g^{0}\right)\right\|_{\operatorname{Str} \times L_{t}^{\infty}}, \\
\partial_{t}\left\langle\mathcal{L} \eta^{0} \mid P_{\gamma} \eta^{0}\right\rangle / 2= & B\left(w^{0}, c^{0}\right)\left[\left\langle\nabla f^{\prime}(Q) \eta_{1}^{0} \mid \nabla \eta^{0}\right\rangle-\left\langle\mathcal{L} \eta^{0} \mid P_{d} \nabla \eta^{0}\right\rangle\right] \\
& +\left\langle\mathcal{L} P_{\gamma} \eta^{0}\right|\left(0, g^{0} \cdot \nabla f^{\prime}(Q) v_{1}^{0}\right) \\
& \left.+\tau_{c^{0}}^{*}\left[\partial_{(w, c)} \widehat{F}\left(w^{0}, c^{0}\right) \cdot\left(z^{0}, g^{0}\right)\right]\right\rangle,
\end{aligned}
$$

and so using (6.36) we obtain

$$
\left\|\eta_{\geq 0}^{0}(0)\right\|_{E} \leq(1+C \kappa+C \sqrt{\delta}+C \ell)\|\psi\|_{E}+C|q|(\delta+\ell) .
$$

Also using (6.30)-(6.36), we have

$$
\begin{aligned}
\left\|\mathcal{R} v^{0}\right\|_{E} & \lesssim\left|g^{0}\right|\left\|v^{0}\right\|_{E} \lesssim \delta\left(\|\psi\|_{E}+|q|\right), \\
\phi_{\delta}\left(\left\|v_{\geq 0}^{1}\right\|_{E}\right) & \leq(1+C \kappa+C \delta+C \ell) \phi_{\delta}\left(\left\|\varphi^{1}\right\|_{E}\right), \\
\left|\triangleleft c^{\triangleright}(0)\right| & \leq|b|+C\left\|\triangleleft B\left(w^{\triangleright}, c^{\triangleright}\right)\right\|_{L_{t}^{\infty}} \leq|b|+C \delta\left[\left\|\varphi^{0}-\tau_{b} \varphi^{1}\right\|_{E}+|b|\right], \\
\left|g^{0}\right| & \leq|q|+C\left\|B_{w}\left(w^{0}, c^{0}\right) z^{0}+B_{c}\left(w^{0}, c^{0}\right) g^{0}\right\|_{L_{t}^{\infty}} \\
& \leq|q|+C \delta\left[\|\psi\|_{E}+|q|\right] .
\end{aligned}
$$

Putting (6.53), (6.55), and the above estimates together, and using $\kappa+\sqrt{\delta}+\ell \ll \underline{k}$, we see that the leading term in (6.43) is bounded by

$$
\frac{1}{2} \Lambda\left[\left\|\varphi^{0}-\tau_{b} \varphi^{1}\right\|_{E}+|b| \phi_{\delta}\left(\left\|\varphi^{1}\right\|_{E}\right)\right]^{\alpha, 1}\left[\|\psi\|_{E}+|q| \phi_{\delta}\left(\left\|\varphi^{1}\right\|_{E}\right)\right]
$$

so the remaining half can absorb the negligible terms, concluding the proof in the nonlinear case $\left\|\varphi^{\triangleright}\right\|_{E} \lesssim \delta$.

Mobile-differentiability of the fixed point $G_{*} \in \mathcal{G}_{\ell, \delta}$ now follows from the closedness of $\mathcal{G}_{\ell, \delta}^{\Lambda}$ for pointwise convergence.

Lemma 6.4. Under the assumption of the above lemma, let $G_{n} \in \mathcal{G}_{\ell, \delta}^{\alpha, \Lambda}$ be a sequence of maps such that $G_{n}(\varphi) \rightarrow G(\varphi)$ as $n \rightarrow \infty$ for all $\varphi \in \mathcal{H}$. Then $G \in \mathcal{G}_{\ell, \delta}^{\alpha, \Lambda}$.

Proof. Since $G_{n} \in \mathcal{G}_{\ell, \delta},(6.4)$ implies that $\mathscr{D} G_{n}(\varphi)$ for each $\varphi \in \mathcal{H}$ is bounded in $\left(\mathcal{H} \times \mathbb{R}^{d}\right)^{*}$. Hence after extracting a subsequence, we have weak convergence of $\mathscr{D} G_{n}(\varphi)$ in $\left(\mathcal{H} \times \mathbb{R}^{d}\right)^{*}$ for $\varphi$ in a dense countable subset $A \subset \mathcal{H}$. To extend the convergence to all $\varphi \in \mathcal{H}$, take a sequence $\varphi_{n}$ converging to $\varphi$ in $\mathcal{H}$. Then for any $\psi \in \mathcal{H}$ and $q \in \mathbb{R}^{d}$, we have

$$
\begin{aligned}
& \left\|\mathscr{D} G_{k}\left(\varphi_{n}\right)(\psi, q)-\mathscr{D} G_{k}\left(\varphi_{m}\right)(\psi, q)\right\|_{E} \\
& \quad \leq \Lambda\left\|\varphi_{n}-\varphi_{m}\right\|^{\alpha, 1}\left[\|\psi\|_{E}+|q| \phi_{\delta}\left(\left\|\varphi_{n}\right\|_{E}\right)\right] \rightarrow 0,
\end{aligned}
$$

as $n, m \rightarrow \infty$, uniformly for all $k \in \mathbb{N}$. Hence we have the convergence

$$
\lim _{k \rightarrow \infty} \mathscr{D} G_{k}(\varphi)=\lim _{k \rightarrow \infty} \lim _{n \rightarrow \infty} \mathscr{D} G_{k}\left(\varphi_{n}\right)=\lim _{n \rightarrow \infty} \lim _{k \rightarrow \infty} \mathscr{D} G_{k}\left(\varphi_{n}\right)
$$

weakly in $\left(\mathcal{H} \times \mathbb{R}^{d}\right)^{*}$. To see the mobile differentiability of $G$, we use the mean value theorem. For any $\varphi, \psi \in \mathcal{H}, q \in \mathbb{R}^{d}, k \in \mathbb{N}$, and $\varepsilon \in \mathbb{R}$ small, there is $\theta \in[0,1]$ such 
that

$$
G_{k}\left(\varphi_{(\varepsilon)}\right)-G_{k}(\varphi)=\varepsilon \mathscr{D} G_{k}\left(\varphi^{\theta \varepsilon}\right)(\psi, q) .
$$

Since $G_{k} \in \mathcal{G}_{\ell, \delta}^{\alpha, \Lambda}$, we have

$$
\begin{aligned}
& \left\|\mathscr{D} G_{k}\left(\varphi^{\theta \varepsilon}\right)(\psi, q)-\mathscr{D} G_{k}(\varphi)(\psi, q)\right\|_{\mathcal{H}} \\
& \quad \lesssim \Lambda\left\|\varphi^{\theta \varepsilon}-\varphi\right\|_{\mathcal{H}}^{\alpha, 1}\left[\|\psi\|_{\mathcal{H}}+|q| \phi_{\delta}\left(\|\varphi\|_{E}\right)\right] \rightarrow 0
\end{aligned}
$$

as $\varepsilon \rightarrow 0$, uniformly for all $k \in \mathbb{N}$. Hence the limit $G(\varphi)$ is mobile differentiable and

$$
\mathscr{D} G(\varphi)(\psi, q)=\lim _{k \rightarrow \infty} \mathscr{D} G_{k}(\varphi)(\psi, q),
$$

which implies $G \in \mathcal{G}_{\ell, \delta}^{\alpha, \Lambda}$.

\begin{tabular}{|c|c|c|}
\hline $\begin{array}{l}\bigcirc^{\triangleright}, \triangleleft \bigcirc^{\triangleright} \\
(\bigcirc \wedge \delta) \\
\bigcirc^{\alpha, 1} \\
B_{R}(\bigcirc) \\
D, J, \mathcal{D}\end{array}$ & $\begin{array}{l}\text { ordered pair and difference } \\
\text { minimum } \\
\text { sum of two powers } \\
\text { a ball in the Banach space } \\
\text { basic operators }\end{array}$ & $\begin{array}{l}(2.1) \\
(2.3) \\
(6.7) \\
(5.1) \\
(1.15),(2.7),(2.9)\end{array}$ \\
\hline$f, d, p$ & nonlinearity, dimension, and power & $(1.6),(1.7)$ \\
\hline$N(v), \vec{N}(v), N_{c}(w), C(v)$ & higher-order nonlinearity around $Q$ & $(2.20),(2.21),(3.9),(5.9)$ \\
\hline$A(v), A_{c}(w)$ & transport terms & $(2.26),(3.9)$ \\
\hline$\chi_{\delta}(v)$ & localizer around 0 in $\mathcal{H}$ & $(3.1)$ \\
\hline$M(v), M_{\delta}(v)$ & nonlinearity for $v$ & $(2.27),(3.2)$ \\
\hline$B(w, c), F(w, c)$ & nonlinearity for $(\mathrm{c}, \mathrm{w})$ & $(3.8)$ \\
\hline$U(t), \mathcal{U}(t)$ & localized flow in $\mathcal{H}$ and on graphs & (3.18), Lemma 3.4 \\
\hline$E(u), P(u)$ & energy and momentum & $(1.3),(2.22)$ \\
\hline $\mathcal{H}, \mathcal{H}_{0}, \mathcal{H}_{\perp}$ & energy space and its subsets & $(1.4),(2.23),(2.24)$ \\
\hline$\|\cdot\|_{E}, \kappa$ & energy norm and the parameter & $(2.18),(2.17)$ \\
\hline$\langle\cdot \mid \cdot\rangle,\langle\cdot, \cdot\rangle_{\mathcal{H}}, \omega(\cdot, \cdot)$ & bilinear forms on $\mathcal{H}$ & $(2.4),(2.5),(2.6)$ \\
\hline$\|\cdot\|_{\text {Str }}$ & Strichartz norm & $(3.10)$ \\
\hline$Q, Q_{c}, Q(p, q)$ & the static solution, its transforms, & $(1.9),(1.12),(1.10)$ \\
\hline$\vec{Q}, \vec{Q}(p, q), \mathscr{S}(Q), \mathscr{S}_{0}(Q)$ & vector forms, and the families & $(1.13),(1.14),(5.18)$ \\
\hline$H(Q)=H_{\alpha \beta}(Q)$ & kinetic energy matrix of $Q$ & $(2.14)$ \\
\hline$L_{+}, \mathcal{L}$ & linearized operators at $Q$ & $(1.15),(2.8)$ \\
\hline$k, K, \underline{k}, \bar{k}$ & eigenvalues and their bounds & $(2.11)$ \\
\hline$\rho_{k}, g_{k \pm}$ & eigenfunctions of $L_{+}, \mathcal{L}$ & $(2.12),(2.13)$ \\
\hline$\lambda_{k \pm}, \mu, \nu, \gamma, v_{ \pm}, v_{d}, \ldots$ & spectral components of $v$ & $(2.15),(2.16)$ \\
\hline$P_{ \pm}, P_{0}, P_{d}, P_{\mu}, P_{\gamma}, \ldots$ & the corresponding operators & $(2.15),(2.16)$ \\
\hline $\begin{array}{l}\mathfrak{m}_{\phi}, \tilde{\mathfrak{m}}_{\delta}, \phi_{\delta} \\
\tau_{c}, \tau^{j}\end{array}$ & $\begin{array}{l}\text { mobile distances and the cutoff } \\
\text { translation operators }\end{array}$ & $\begin{array}{l}(2.28),(2.38),(2.39) \\
(3.6),(3.28)\end{array}$ \\
\hline $\mathcal{G}_{\ell, \delta}, \mathcal{G}, \mathcal{G}_{\ell, \delta}^{\alpha, \Lambda}$ & sets of Lipschitz maps & $(3.53),(3.69)$, Definition 6.2 \\
\hline$\lceil G\rfloor$ & the graph in $\mathcal{H}$ & $(3.54)$ \\
\hline$G_{*}$ & the invariant map & Theorem 3.6 \\
\hline$\widetilde{G}$ & projection to $\mathcal{H}_{\perp}$ & Lemma 5.1 \\
\hline $\mathscr{D Q G}$ & mobile derivative & Definition 6.1 \\
\hline$\varphi_{(\varepsilon)}(\varepsilon)$ & translating variation in $\mathcal{H}$ & Definition 6.1 \\
\hline$\widehat{G}_{t}$ & complemented graphs & $(6.8)$ \\
\hline $\mathcal{R}$ & spectral error of the $g$-translation & $(6.28)$ \\
\hline
\end{tabular}

Therefore the fixed point $G_{*}$ belongs to $\mathcal{G}_{\ell, \delta}^{\alpha, \Lambda}$ and so in particular $C^{1, \alpha}$ in the $\mathcal{H}$ topology. Then it is easy to see that $\widetilde{G}_{*}$ is also $C^{1, \alpha}$, as are $\mathcal{M}_{c u, 0}$ and $\mathcal{M}_{c u}$.

\section{Appendix A. Table of notation.}

Copyright (C) by SIAM. Unauthorized reproduction of this article is prohibited. 


\section{REFERENCES}

[1] P. W. Bates and C. K. R. T. Jones, Invariant Manifolds for Semilinear Partial Differential Equations, Dynam. Report. Ser. Dynam. Systems Appl. 2, Wiley, Chichester, UK, 1989, pp. 1-38.

[2] P. Bates, K. Lu, and C. Zeng, Approximately invariant manifolds and global dynamics of spike states, Invent. Math., 174 (2008), pp. 355-433.

[3] P. Bates, K. Lu, And C. Zeng, Existence and persistence of invariant manifolds for semiflows in Banach space, Mem. Amer. Math. Soc., 135 (1998).

[4] M. Beceanu, New estimates for a time-dependent Schrödinger equation, Duke Math. J., to appear.

[5] M. Beceanu, A critical center-stable manifold for Schrödinger equation in three dimensions, Comm. Pure Appl. Math., 65 (2012), pp. 431-507.

[6] H. Berestycki And P.-L. Lions, Nonlinear scalar field equations. I. Existence of a ground state, Arch. Ration. Mech. Anal., 82 (1983), pp. 313-345.

[7] H. Berestycki And P.-L. Lions, Nonlinear scalar field equations. II. Existence of infinitely many solutions, Arch. Ration. Mech. Anal., 82 (1983), pp. 347-375.

[8] V. Buslaev and G. Perelman, Scattering for the nonlinear Schrödinger equation: States that are close to a soliton, St. Petersburg Math. J., 4 (1993), pp. 1111-1142.

[9] V. Buslaev and G. Perelman, Nonlinear scattering: States that are close to a soliton, J. Math. Sci., 77 (1995), pp. 3161-3169.

[10] O. Costin, M. Huang, And W. Schlag, On the spectral properties of $L_{ \pm}$in three dimensions, Nonlinearity, 25 (2012), pp. 125-164.

[11] S. Cuccagna, On Asymptotic Stability of Moving Ground States of the Nonlinear Schrödinger Equation, preprint, 2011.

[12] L. Demanet and W. Schlag, Numerical verification of a gap condition for a linearized nonlinear Schrödinger equation, Nonlinearity, 19 (2006), pp. 829-852.

[13] G. Fibich, F. Merle, And P. RAPhaËL, Proof of a spectral property related to the singularity formation for the $L^{2}$ critical nonlinear Schrödinger equation, Phys. D, 220 (2006), pp. 113.

[14] F. Gesztesy, C. K. R. T. Jones, Y. Latushkin, and M. Stanislavova, A spectral mapping theorem and invariant manifolds for nonlinear Schrödinger equations, Indiana Univ. Math. J., 49 (2000), pp. 221-243.

[15] J. Krieger and W. Schlag, Stable manifolds for all monic supercritical focusing nonlinear Schrödinger equations in one dimension, J. Amer. Math. Soc., 19 (2006), pp. 815-920.

[16] J. Marzuola and G. Simpson, Spectral analysis for matrix Hamiltonian operators, Nonlinearity, 24 (2011), pp. 389-429.

[17] K. NAKANishi AND W. Schlag, Global dynamics above the ground state energy for the focusing nonlinear Klein-Gordon equation, J. Differential Equations, 250 (2011), pp. 2299-2333.

[18] K. NAKANishi AND W. Schlag, Global dynamics above the ground state for the nonlinear KleinGordon equation without a radial assumption, Arch. Ration. Mech. Anal., 203 (2012), pp. 809-851.

[19] K. NAKAnishi And W. Schlag, Invariant Manifolds and Dispersive Hamiltonian Evolution Equations, Zürich Lectures in Advanced Math., European Mathematical Society, Zurich, 2011.

[20] G. Perelman, Two soliton collision for nonlinear Schrödinger equations in dimension 1, Ann. Inst. H. Poincaré Anal. Non Linéaire, 28 (2011), pp. 357-384.

[21] G. Perelman, On the formation of singularities in solutions of the critical nonlinear Schrödinger equation, Ann. Henri Poincaré, 2 (2001), pp. 605-673.

[22] W. SCHLAG, Stable manifolds for an orbitally unstable nonlinear Schrödinger equation, Ann. of Math. (2), 169 (2009), pp. 139-227.

[23] M. Weinstein, Modulational stability of ground states of nonlinear Schrödinger equations, SIAM J. Math. Anal., 16 (1985), pp. 472-491.

Copyright (C) by SIAM. Unauthorized reproduction of this article is prohibited. 